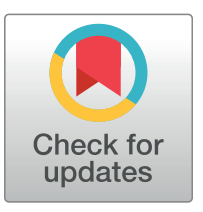

\section{G OPEnACCESS}

Citation: Wang J, Chan B, Tong M, Paung Y, Jo U, Martin D, et al. (2019) Prolyl isomerization of FAAP20 catalyzed by PIN1 regulates the Fanconi anemia pathway. PLoS Genet 15(2): e1007983. https://doi.org/10.1371/journal.pgen.1007983

Editor: Gregory S. Barsh, Stanford University School of Medicine, UNITED STATES

Received: August 13, 2018

Accepted: January 23, 2019

Published: February 21, 2019

Copyright: ๑ 2019 Wang et al. This is an open access article distributed under the terms of the Creative Commons Attribution License, which permits unrestricted use, distribution, and reproduction in any medium, provided the original author and source are credited.

Data Availability Statement: All relevant data are within the manuscript and its Supporting Information files.

Funding: This work was supported by: The US National Institute of Health (R01CA218132) (to $\mathrm{H}$. K); Alexandrine and Alexander L. Sinsheimer Foundation (to H.K.); The Research Foundation at SUNY Stony Brook and the Cancer Center (to H.K); Scholars in Biomedical Sciences (to J.W.) and The US National Institute of Health (R35GM119437) (to M.S.). The funders had no role in study design,
RESEARCH ARTICLE

\section{Prolyl isomerization of FAAP20 catalyzed by PIN1 regulates the Fanconi anemia pathway}

\author{
Jingming Wang ${ }^{1}$, Bryan Chan $\odot^{1}$, Michael Tong ${ }^{1}$, YiTing Paung ${ }^{1,2}$, Ukhyun Jo ${ }^{11}$, \\ Dwight Martin $\oplus^{3}$, Markus Seeliger $\oplus^{1,4}$, John Haley $\oplus^{3,4}$, Hyungjin Kim $\varpi^{1,4 *}$
}

1 Department of Pharmacological Sciences, Stony Brook University, Stony Brook, New York, United States of America, 2 Department of Chemistry, Stony Brook University, Stony Brook, New York, United States of America, 3 Department of Pathology, Proteomics Center, Stony Brook University, Stony Brook, New York, United States of America, 4 Stony Brook Cancer Center, Stony Brook University School of Medicine, Stony Brook, New York, United States of America

a Current address: Developmental Therapeutics Branch and Laboratory of Molecular Pharmacology, National Cancer Institute, NIH, Bethesda, Maryland, United States of America

* Hyungjin.kim@stonybrook.edu

\section{Abstract}

The Fanconi Anemia (FA) pathway is a multi-step DNA repair process at stalled replication forks in response to DNA interstrand cross-links (ICLs). Pathological mutation of key FA genes leads to the inherited disorder FA, characterized by progressive bone marrow failure and cancer predisposition. The study of FA is of great importance not only to children suffering from FA but also as a model to study cancer pathogenesis in light of genome instability among the general population. FANCD2 monoubiquitination by the FA core complex is an essential gateway that connects upstream DNA damage signaling to enzymatic steps of repair. FAAP20 is a key component of the FA core complex, and regulated proteolysis of FAAP20 mediated by the ubiquitin $\mathrm{E} 3$ ligase $\mathrm{SCF}^{\mathrm{FBW} 7}$ is critical for maintaining the integrity of the FA complex and FA pathway signaling. However, upstream regulatory mechanisms that govern this signaling remain unclear. Here, we show that PIN1, a phosphorylation-specific prolyl isomerase, regulates the integrity of the FA core complex, thus FA pathway activation. We demonstrate that PIN1 catalyzes cis-trans isomerization of the FAAP20 pSer48Pro49 motif and promotes FAAP20 stability. Mechanistically, PIN1-induced conformational change of FAAP20 enhances its interaction with the PP2A phosphatase to counteract $\mathrm{SCF}^{\mathrm{FBW}}$-dependent proteolytic signaling at the phosphorylated degron motif. Accordingly, PIN1 deficiency impairs FANCD2 activation and the DNA ICL repair process. Together, our study establishes PIN1-dependent prolyl isomerization as a new regulator of the FA pathway and genomic integrity.

\section{Author summary}

Fanconi anemia (FA) is a devastating disease of children that leads to birth defects, bone marrow failure, and a variety of cancers early in their lives. Germ-line mutations in FA genes disrupt the DNA repair process, namely the FA pathway, resulting in genome instability and clinical features of FA patients. Thus, understanding the molecular mechanisms 
data collection and analysis, decision to publish, or preparation of the manuscript.

Competing interests: The authors have declared that no competing interests exist. by which the FA pathway is regulated is critical for alleviating the burden of children suffering from FA and related cancer. A critical step in this pathway is the monoubiquitination of FANCD2 by a multi-subunit ubiquitin E3 ligase called the FA core complex, and the FAAP20 subunit is required for its functional integrity. Here, we show that prolinedirected structural change of FAAP20 catalyzed by the PIN1 prolyl cis-trans isomerase is essential for the FAAP20 stability by counteracting phosphorylation-dependent proteolytic signaling of FAAP20 and thus promotes FANCD2 activation and DNA repair. Our findings reveal how PIN1-mediated phosphorylation signaling cascade and proteolysis preserves genomic integrity and how its deregulation is associated the pathogenesis of FA. Our knowledge on a new regulatory mechanism governing FA pathway activation may lead to the development of a new target for FA and FA-related malignancy.

\section{Introduction}

The Fanconi anemia (FA) DNA repair pathway resolves DNA interstrand cross-links (ICLs) and other obstacles encountered during DNA replication [1,2]. Germ-line mutation of at least 22 genes involved in this pathway not only causes a childhood blood disorder of bone marrow failure, FA, but also predisposes affected children to a variety of cancers, highlighting the role of the FA pathway as a tumor suppressor mechanism that preserves genomic integrity $[3,4]$. Central to this pathway is FANCD2 activation, triggered by its monoubiquitination via a multi-subunit ubiquitin E3 ligase, the FA core complex, which targets the FANCD2-FANCI heterodimeric complex to DNA lesions to recruit structure-specific nucleases and initiate nucleolytic incision of cross-linked DNA [5,6]. Thus, FANCD2 monoubiquitination by the FA core complex constitutes an essential gateway to connect the DNA damage response (DDR) to enzymatic steps of DNA ICL repair. Monoubiquitinated FANCD2 is also required for maintaining DNA replication fork integrity independently of DNA ICL processing $[7,8]$. The FA core complex consists of at least eight FA gene products associated with several accessory proteins and exhibits modular features to promote the activity of the catalytic E3 ligase core [911]. Each subunit is under the control of numerous posttranslational modifications, implicating multiple layers of regulation in response to DNA damage and replication checkpoint [12]. The FANCA-FANCG-FAAP20 subcomplex constitutes a structural module to maintain the integrity of the FA complex and supports its localization to the sites of DNA lesions $[10,11]$. The $20 \mathrm{kD}$ FA-associated protein FAAP20 directly interacts with FANCA and promotes its stability [13-15]. In the absence of FAAP20, the degron motif of FANCA is exposed to undergo SUMO-dependent proteolytic degradation, leading to the loss of the FA core complex integrity and thus a defect in FANCD2 monoubiquitination [16]. Accordingly, a patientderived mutation that disrupts the FANCA-FAAP20 interaction causes FA-like phenotypes [16]. The dynamics of the FANCA-FAAP20 interaction is regulated by FAAP20 degradation, which is mediated by the SKP1-CUL1-F-Box/FBW7 $\left(\mathrm{SCF}^{\mathrm{FBW} 7}\right)$ ubiquitin E3 ligase complex [17]. Specifically, glycogen synthase kinase $3 \beta$ (GSK3 $\beta$ )-dependent phosphorylation of FAAP20 at the Cdc4 phospho-degron (CPD) motif is recognized by FBW7 to trigger polyubiquitination and proteasome-dependent FAAP20 degradation [17]. Hence, phosphorylationdependent ubiquitin signaling plays an essential role in regulating the FANCA-FAAP20 interaction and FA pathway activation. Nevertheless, the upstream signaling that governs FAAP20 phosphorylation status and its detailed mode of action for FAAP20 degradation remain uncharacterized. 
The reversible phosphorylation-dependent ubiquitin-proteasome system (UPS) is a fundamental regulatory mechanism for protein degradation. As exemplified by FBW7-dependent FAAP20 degradation, phosphorylation of the phospho-degron motif allows proteins to be recognized by a ubiquitin E3 ligase and delivered to the proteasome. Meanwhile, given the rapid phosphorylation-dephosphorylation event mediated by kinases and phosphatases, catalysis by peptidyl-prolyl cis-trans isomerase NIMA-interacting 1 (PIN1) is often considered a key ratedetermining step in controlling phosphorylation-dependent signaling [18]. PIN1 specifically recognizes a phosphorylated Ser or Thr residue preceding a Pro (pSer/Thr-Pro). By catalyzing the Pro cis-trans isomerization that converts substrates into a conformation that is favorable or refractory to downstream signaling, PIN1 acts as a molecular switch to control diverse cellular functions, including proteolysis [19]. Accordingly, previous studies have established the role of PIN1 in regulating the stability of oncoproteins and tumor suppressors in multiple cellular processes, including transcriptional regulation (c-Jun, c-Myc, p53), cell cycle (Cyclin D1 \& E), and cell death (MCL-1) [20-27]. Notably, many of these substrates are also substrates for $\mathrm{SCF}^{\mathrm{FBW}}$, implying a complex interplay at the PIN1-SCF ${ }^{\mathrm{FBW} 7}$ phospho-dependent ubiquitin signaling axis to modulate substrate ubiquitination and degradation. Interestingly, a recent study has revealed the role of PIN1 in promoting degradation of CtIP, a mediator of doublestrand break (DSB) repair, thereby connecting PIN1 signaling to DNA repair processes [28]. However, the molecular details of how PIN1 regulates its substrates associated with DNA repair are only beginning to be understood.

Here, we identify PIN1 as a new regulator of the FA pathway. We provide evidence that FAAP20 is a new substrate of PIN1 and that PIN1 antagonizes proteolytic signaling of FAAP20 degradation mediated by $\mathrm{SCF}^{\mathrm{FBW}}$, thus promoting the integrity of the FA core complex and FANCD2 activation. Together, our study uncovers a new role for the prolyl isomerase PIN1 in governing the DNA ICL repair process and genomic integrity. Given that PIN1 is deregulated in many human cancers, our findings also provide insights into how the disruption of FA pathway signaling may be connected to the genome instability of PIN1-related cancers.

\section{Results}

\section{The FANCA binding-defective FAAP20 mutant is prone to prolyl isomerization}

FANCA and FAAP20 interact and stabilize each other in the FA core complex $[13,15,16]$. We and others have previously shown that the N-terminal region of FAAP20 is required for the FANCA interaction (Fig 1A) [13,14]. While further charactering the FAAP20-FANCA interaction via extensive mutagenesis, we serendipitously found that one FAAP20 mutant (W40A, L44Q, R45A; hereinafter WLR), which fails to interact with FANCA, exhibits an additional, slower-migrating upper isoform during SDS-PAGE (Fig 1B; lane 8). This additional band was specific to the WLR mutant, and not seen with the CPD degron mutant (Fig 1C). This drastic mobility shift may reflect a conformational change of the proline backbone, which often persists even under denaturing conditions, as previously seen in the PIN1 substrate ATR [29]. Notably, mass spectrometry analysis of both Flag-FAAP20 WLR isoforms revealed that the upper form is phosphorylated at Ser48 adjacent to Pro49 (S1A-1C Fig). Since the phosphorylated Ser-Pro motif is known to be a target for PIN1-catalyzed cis-trans isomerization, we determined whether pS48-P49 is responsible for the structural change of the FAAP20 WLR mutant. Indeed, mutations either in the phosphorylated residue Ser48 or in the isomerizing residue Pro49 abolished the upper form (Fig 1D). Overexpression of PIN1 increased the ratio 
A

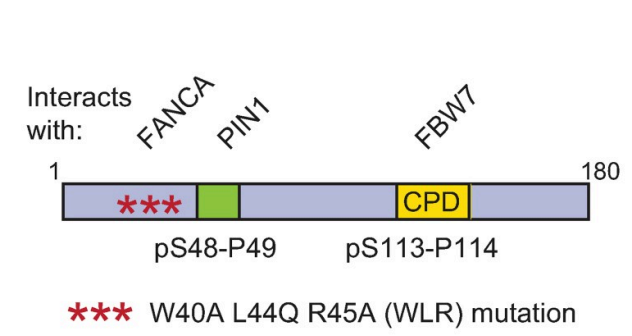

C

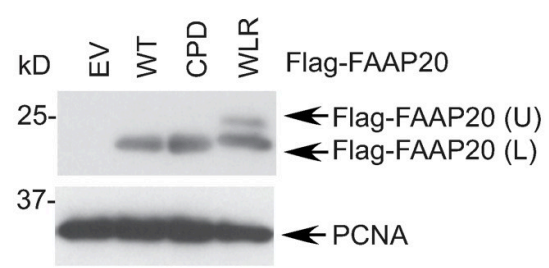

E

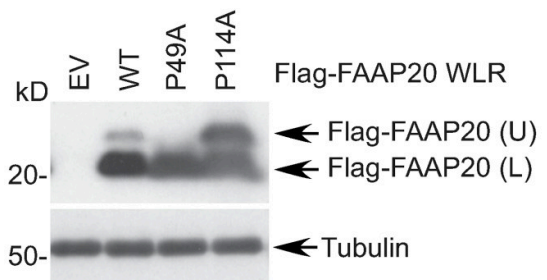

B

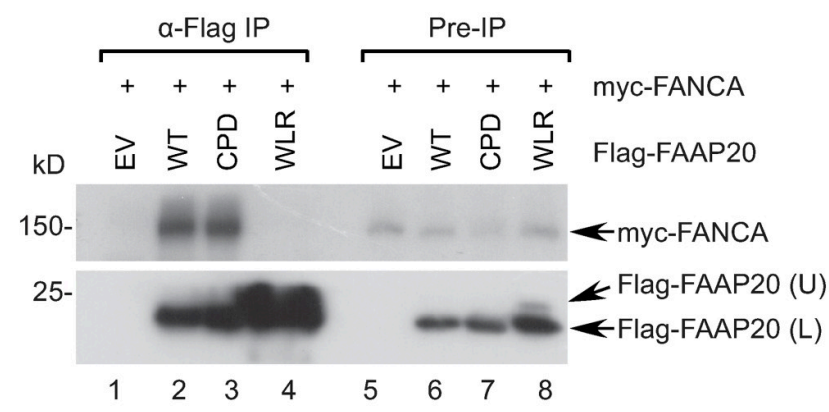

D

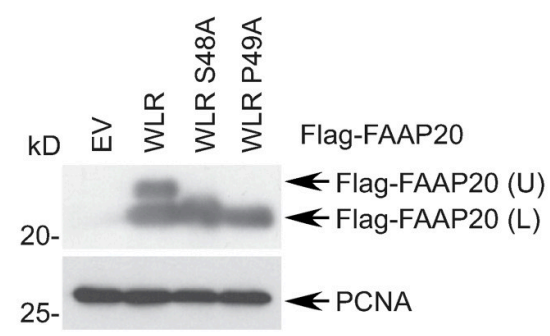

F

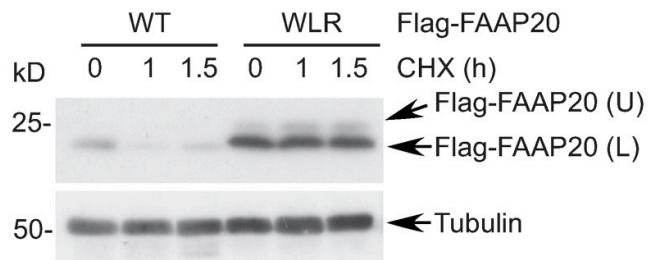

Fig 1. The FANCA binding-defective FAAP20 mutant is isomerized at the pS48-P49 motif. (A) A schematic of the FAAP20 structure, displaying the pS48-P49 motif for PIN1 binding and the pS113-P114 CPD phospho-degron motif for FBW7 binding. The Nterminal region of FAAP20 mediates its interaction with FANCA, which is disrupted by W40A, L44Q, and R45A (WLR) mutations. (B) 293 T cells transiently transfected with indicated plasmids were subjected to anti-Flag immunoprecipitation (IP) and Western blotting (WB). CPD: S113A \& S117A mutation. The slower migrating Flag-FAAP20 isoform is designated as (U: upper) in contrast to the faster migrating isoform (L: lower). WB analysis of U2OS cells expressing Flag-FAAP20 wild-type (WT) or various mutants. EV: empty vector. (C-E) 293T cells transiently transfected with indicated plasmids were analyzed by WB against anti-Flag and anti-PCNA antibodies. (F) U2OS cells transiently expressing Flag-FAAP20 WT or WLR mutant were treated with $50 \mu \mathrm{g} / \mathrm{mL}$ cycloheximide (CHX) for the indicated times, and FAAP20 degradation was monitored by WB.

https://doi.org/10.1371/journal.pgen.1007983.g001

of the upper versus lower form as well as overall FAAP20 WLR levels, while PIN1 knockdown decreased the levels of the upper form (S1D Fig). FAAP20 contains two pSer-Pro motifs, one of which we previously defined as a degron motif that is recognized by FBW7 [17]. Some of the CPD of FBW7 substrates have previously been shown to undergo isomerization by PIN1, thereby directly affecting signaling centered on the CPD [22]. Nevertheless, unlike the Pro49 mutation, disruption of the Pro at the CPD did not abolish the upper form of the FAAP20 WLR (Fig 1E). Interestingly, the FAAP20 WLR mutant was much more stable than wild-type (WT) upon inhibition of nascent protein synthesis by cycloheximide, despite its inability to interact with its protective partner FANCA, suggesting that downstream proteolytic signaling is impaired (Fig 1F). Together, these data indicate that the FAAP20 WLR mutation leads to a structural change specific to the pS48-P49 motif and influences FAAP20 stability. 


\section{FAAP20 interacts with PIN1 in a phosphorylation-dependent manner}

Our data from the FAAP20 WLR mutant raises the possibility that the pS48-P49 motif of WT FAAP20 could be a physiological target of PIN1. Thus, we determined whether FAAP20 interacts with PIN1 through the pS48-P49 motif. To this end, we purified GST-tagged PIN1 from E. coli and incubated with in vitro transcribed and translated (IVTT) Flag-tagged FAAP20. GST-PIN1 directly interacted with Flag-FAAP20 WT, whereas the PIN1 W34A substratebinding mutant [30] failed to do so (Fig 2A). GST-PIN1 also pulled down endogenous FAAP20 from cell lysates (S2A Fig). Similarly, treatment with lambda protein phosphatase also decreased the interaction, arguing for the requirement of FAAP20 phosphorylation for the

A

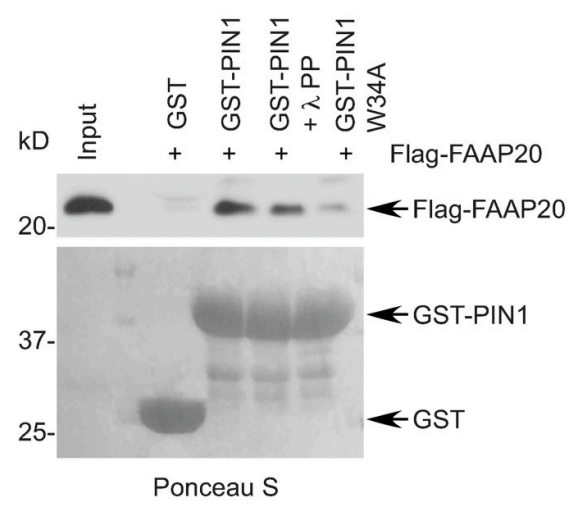

C

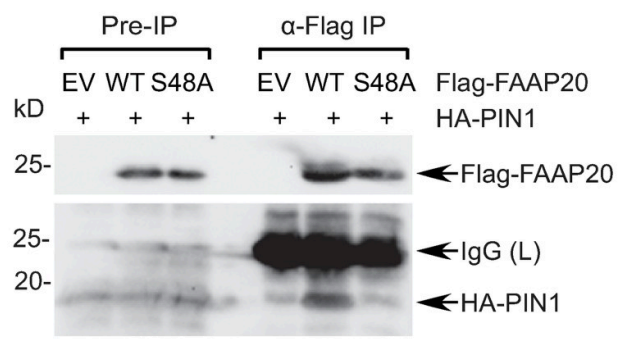

$\mathbf{E}$

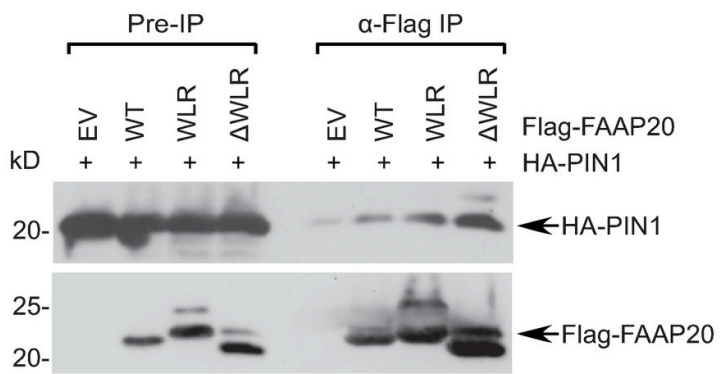

B

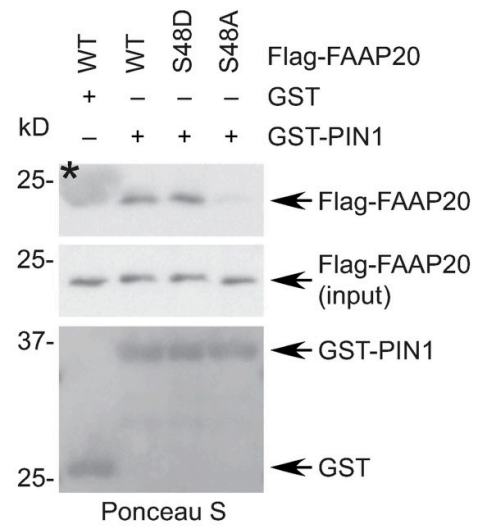

D

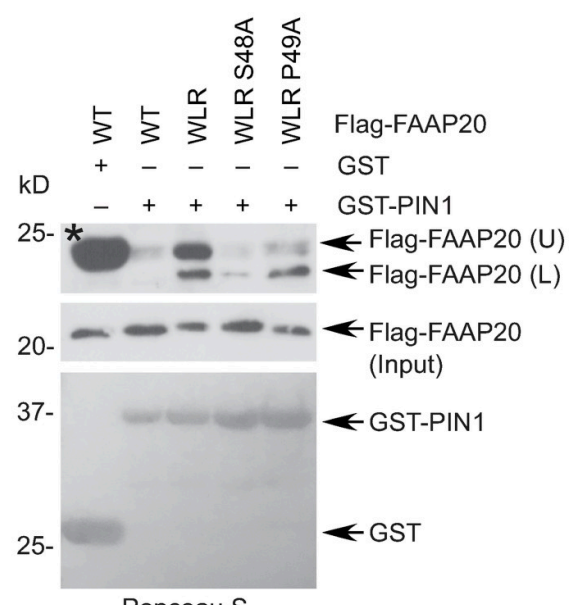

Ponceau S

Fig 2. FAAP20 interacts with PIN1 in a phosphorylation-dependent manner. (A, B) In vitro transcribed and translated (IVTT) FAAP20 WT or mutants were incubated with glutathione beads bound with GST- or GST-PIN1 WT or W34A substratebinding mutant and analyzed by WB. The input for recombinant GST proteins was analyzed by Ponceau S staining. Where indicated, $20 \mathrm{U} / \mu \mathrm{L}$ lambda protein phosphatase $(\lambda \mathrm{PP})$ was incubated at $30{ }^{\circ} \mathrm{C}$ for $30 \mathrm{~min} .{ }^{*}$ denotes GST signal visualized nonspecifically during anti-Flag immunoblots. (C) $293 \mathrm{~T}$ cells transfected with indicated plasmids were subjected to anti-Flag IP and WB. (D) GST pull-down of IVTT FAAP20 WT or WLR mutant variants. Note the appearance of the Flag-FAAP20 (U) isoform upon being pulled down by GST-PIN1. (E) Enhanced interaction between HA-PIN1 and Flag-FAAP20 WLR (W40, L44Q, R45A) or $\Delta$ WLR (a.a.40-45 deletion) revealed by anti-Flag IP and WB of 293T cell lysates.

https://doi.org/10.1371/journal.pgen.1007983.g002 
PIN1 interaction (Fig 2A). Indeed, the non-phosphorylatable FAAP20 S48A mutant was unable to interact with PIN1 in vitro, whereas the WT or the phospho-mimic S48D mutant retained the interaction (Fig 2B). Moreover, Flag-FAAP20 WT, but not S48A, immunoprecipitated HA-PIN1 from cell lysates (Fig 2C). Notably, GST-PIN1 interacted with the WLR mutant stronger than with WT FAAP20 in vitro and induced the formation of the upper form while pulling down the FAAP20 WLR, indicating that enhanced PIN1 interaction renders the FAAP20 WLR more susceptible to the action of PIN1 and subsequent isomerization (Fig 2D). In contrast, the IVTT FAAP20 WLR itself did not exhibit its shifted isoform when it was immunoprecipitated alone in vitro in the absence of PIN1, indicating that the isoform directly results from the structural change induced by PIN1, rather than representing a posttranslational modification that may have occurred during incubation (S2B Fig). We also showed that the FAAP20 WLR point or deletion mutants interact more strongly with PIN1 in the cells in comparison to WT, suggesting that increased affinity of the FAAP20 WLR mutant to PIN1, perhaps due to the change of conformation near the pS48-P49 motif caused by the disruption of the adjacent WLR region, allows enhanced isomerization and appearance of two isoforms (Fig 2E).

\section{PIN1 catalyzes isomerization of the phosphorylated FAAP20 S48-P49 motif}

To further support the idea that FAAP20 is a substrate of PIN1, we monitored the conformational change of FAAP20 catalyzed by PIN1 using NMR spectroscopy. To this end, we synthesized FAAP20 peptides either non-phosphorylated or phosphorylated at Ser48 (Fig 3A). Each peptide was incubated with PIN1, and the cis-trans conformational exchange of the pSer and Glu residues flanking the Pro residue in the peptide was monitored by ${ }^{1} \mathrm{H}-{ }^{-1} \mathrm{H}$ (2D) ROESY (rotating frame Overhause effect spectroscopy) [31]. In this experiment diagonal-peaks corresponding to the amide protons of pSer7 and Glu9 in their cis (cc) and trans (tt) conformations were studied. Cross-peaks that have the same sign as the diagonal peaks indicate conformational exchange between two distinct conformations, but cross-peaks that have the opposite sign indicate an NOE (Nuclear Overhauser effect). In the absence of PIN1, no exchange crosspeaks were detected, suggesting that conformational exchange between the cis and trans conformations was too slow to be detected (Fig 3B, bottom left). In contrast, exchange cross-peaks were observed in the phospho-peptide upon PIN1 incubation, indicating evidence of conformational exchange (Fig 3B, bottom right). As a control, incubation of a non-phosphorylated peptide with PIN1 did not generate any notable exchange cross-peaks (Fig 3B, top). These data suggest that substantially greater conformational exchange occurs in the presence of PIN1, and that PIN1 specifically recognizes the phosphorylated S48-P49 motif of FAAP20 to catalyze its isomerization. We also determined both forward $\left(\mathrm{K}_{\mathrm{ct}}{ }^{\mathrm{cat}}\right)$ and reverse $\left(\mathrm{K}_{\mathrm{tc}}{ }^{\mathrm{cat}}\right)$ rate constants for the two-state conformational exchange process by analyzing the ratio of the $\mathrm{I}_{\mathrm{tc}}$ cross-peak intensity to the $\mathrm{I}_{\mathrm{tt}}$ diagonal-peak intensity (S3A and S3B Fig). Our analysis indicates that the average value of $\mathrm{K}_{\mathrm{ct}}{ }^{\mathrm{cat}}$ is almost 9-fold greater than that of $\mathrm{K}_{\mathrm{tc}}{ }^{\mathrm{cat}}$, which is consistent with previous reports showing that the forward rate of cis-to-trans is greater than the reverse rate of trans-tocis, and that the trans conformation is predominant over the cis conformation (Fig 3C) [32-34].

In addition, we further demonstrated that limited proteolysis of IVTT FAAP20 by trypsin is attenuated when FAAP20 was pre-incubated with recombinant PIN1, indicating that fulllength FAAP20 adapts a different conformation upon PIN1-induced isomerization in vitro (Fig 3D). Together, these data suggest that PIN1 catalyzes the isomerization of FAAP20.

\section{PIN1 activity is required for promoting FAAP20 stability}

PIN1-dependent cis-trans isomerization often exerts a profound impact on the stability of phosphorylated proteins by affecting the ubiquitin signaling required for proteasomal 
A

FAAP20 non-phospho-peptide

ELLRTVS ${ }^{7} P^{8} \mathbf{E}^{9}$ LILD

FAAP20 pS48 phospho-peptide

ELLRTVpS ${ }^{7} P^{8} E^{9} L I L D$

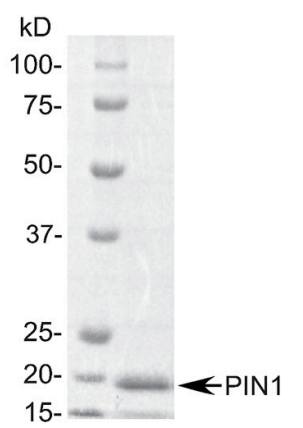

C

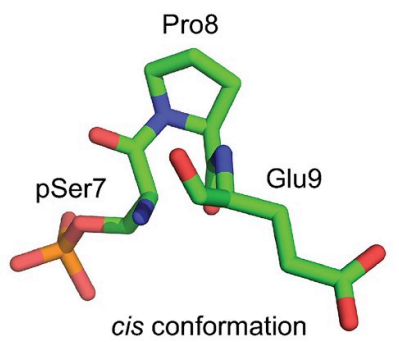

B
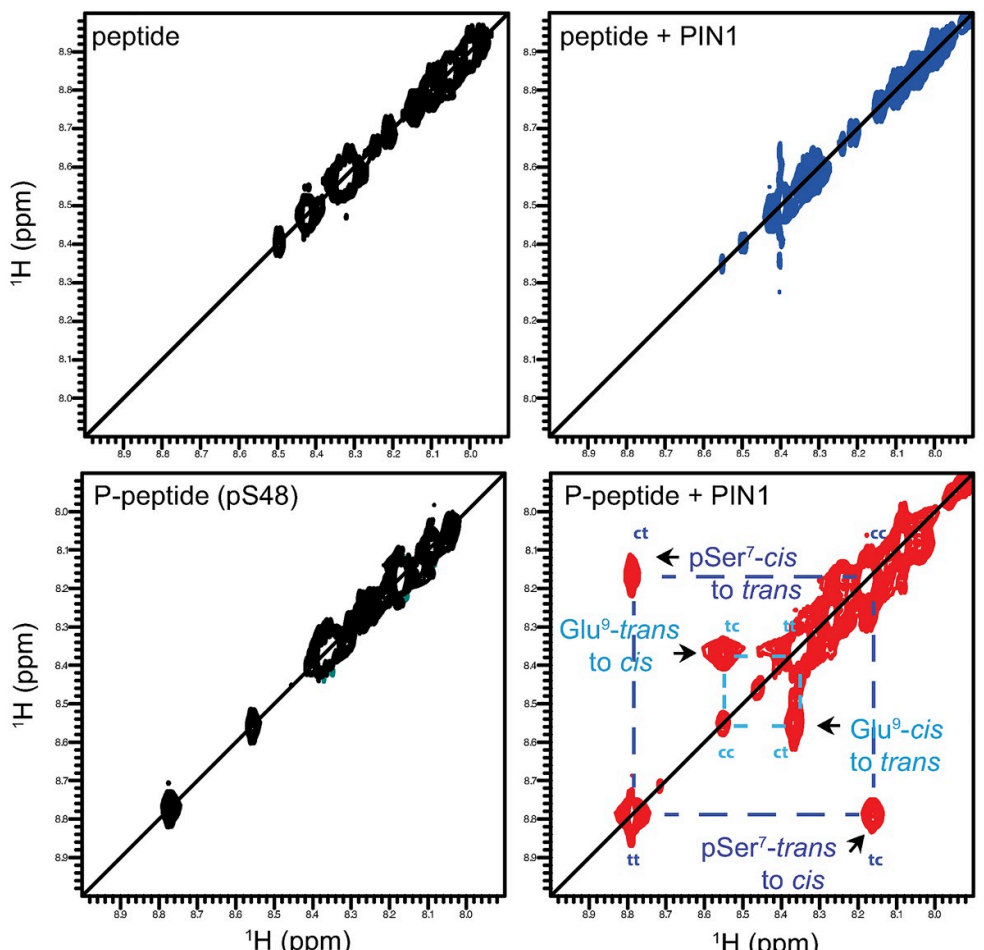

${ }^{1} \mathrm{H}(\mathrm{ppm})$

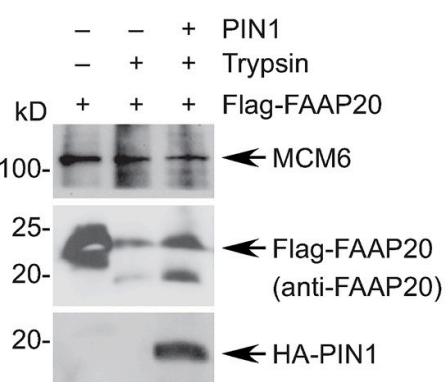

Fig 3. PIN1 catalyzes the isomerization of FAAP20 and induces its conformational change. (A) (Top) The FAAP20 peptides used in NMR study. pSer7 and Glu9, which precedes and follows Pro8, respectively, correspond to the pSer48-Pro49-Glu50 amino acid residues of FAAP20. (Bottom) coomassie blue staining of purified recombinant His-tagged PIN1. (B) 2D ROESY spectra of $2 \mathrm{mM}$ phospho-peptide alone (bottom left) and peptide mixed with $0.03 \mathrm{mM}$ His-PIN1 (bottom right) for $90 \mathrm{~ms}$. Arrows denote cross-peaks representing cis-trans conformational exchange of pSer7 (blue) and Glu9 (pale blue). Dotted lines indicate the amide protons of pSer7 and Glu8 that correlate to the cross-peaks. As a control, a non-phosphorylated peptide was incubated with PIN1 for $300 \mathrm{~ms}$ (top left and right). (C) Illustration of the conformational exchange of the pFAAP20 peptide by PIN1. The isomerization rates of pSer7 reveal an enhanced cis-trans conformational exchange rate by 8.72 -fold. (D) Where indicated, IVTT Flag-FAAP20 was preincubated with $10 \mathrm{ng} / \mu \mathrm{L}$ His-PIN1 followed by $500 \mathrm{ng} / \mathrm{mL}$ trypsin at $30^{\circ} \mathrm{C}$ for $1 \mathrm{~min}$ to undergo limited proteolytic digestion, and the degradation pattern was visualized by anti-FAAP20 WB.

https://doi.org/10.1371/journal.pgen.1007983.g003

degradation [19]. Given that we previously identified FAAP20 as a substrate of $\mathrm{SCF}^{\mathrm{FBW7}}$, which participates in the UPS known to be modulated by PIN1 [30], we determined whether PIN1 affects the stability of FAAP20. Knockdown of PIN1 with two independent siRNAs facilitated the degradation of endogenous FAAP20 upon cycloheximide chase (Fig 4A), and the reduced FAAP20 levels were rescued by proteasome inhibition (Fig 4B), indicating that PIN1 promotes FAAP20 stability in a physiological manner. To further substantiate our findings, we generated PIN1 knockout (KO) U2OS human osteosarcoma cell lines by CRISPR-Cas 9 
A

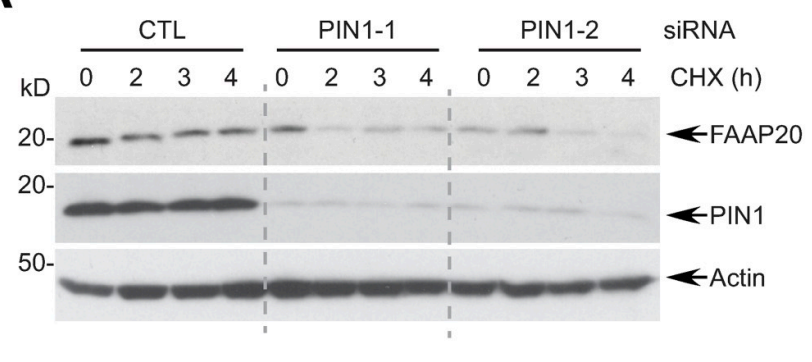

B

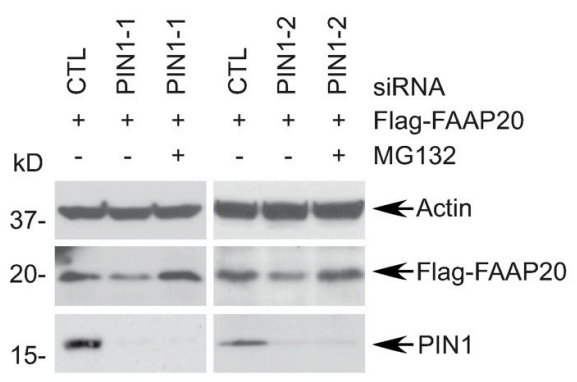

C

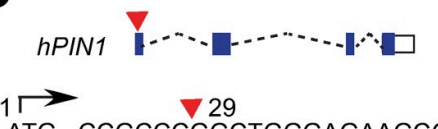

ATG...CCGCCCGGCTGGGAGAAGCGCAT...

...GGCGGGCCGACCCTCTTCGCGTA...

PAM

Target (20 bp)

kD WT \#1 \#6 PIN1-

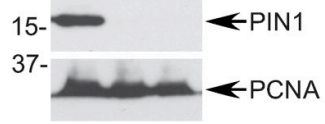

E

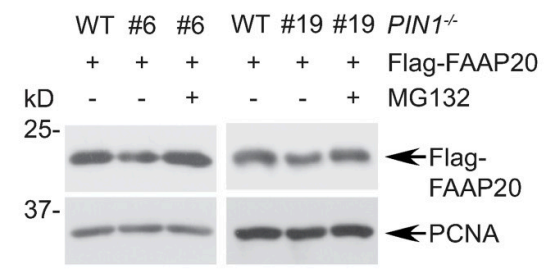

H

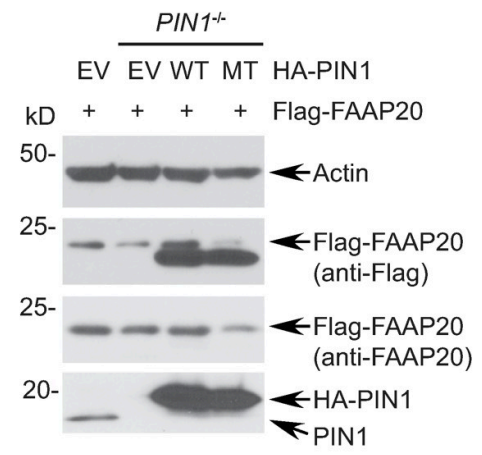

D
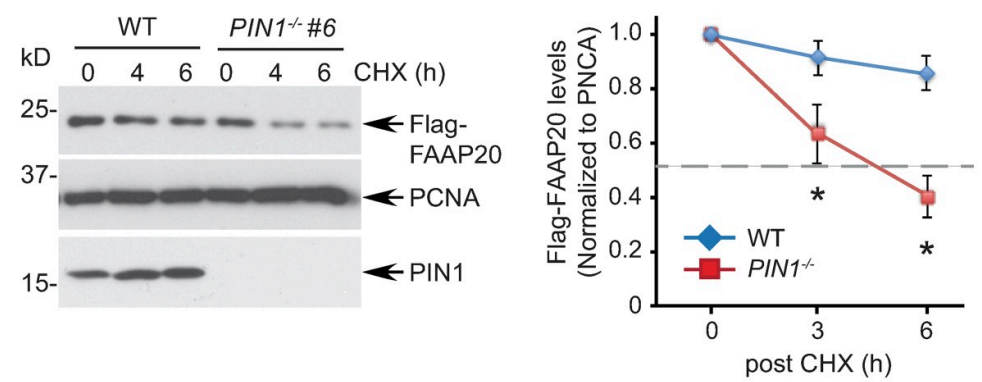

$\mathbf{F}$

G
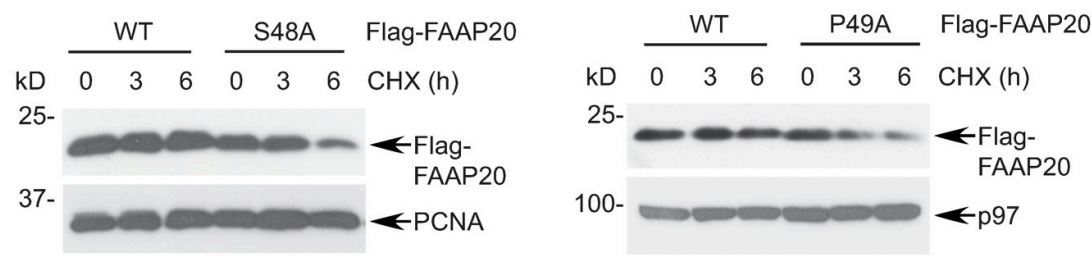

I

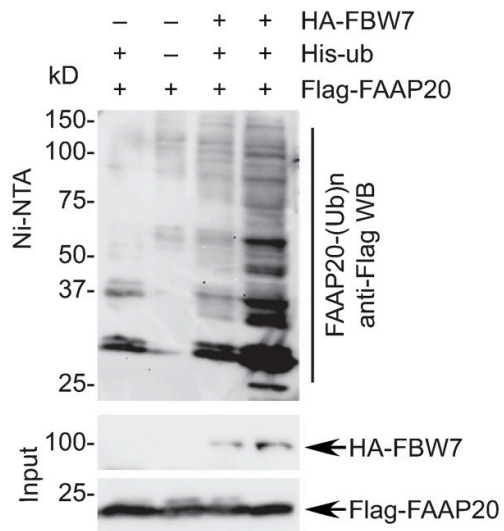

Fig 4. PIN1 is required for maintaining FAAP20 stability. (A) U2OS cells transfected with siRNA control (CTL) or PIN1 were treated with $50 \mu \mathrm{g} / \mathrm{mL}$ CHX for the indicated times, and degradation kinetics of endogenous FAAP20 was monitored by WB. (B) U2OS cells were serially transfected with indicated siRNA oligos and Flag-FAAP20 encoding plasmid, and cell lysates were analyzed by WB. Where indicated, $10 \mu \mathrm{M}$ of proteasome inhibitor MG132 was incubated for $6 \mathrm{~h}$ before harvest. (C) (Top) a schematic for the PIN1 knockout strategy using CRISPR/Cas9. The 20-nucleotide sgRNA target loci on the bottom strand of exon 1 are marked in blue line along with a PAM sequence in red. The cleavage site for the Cas9 nuclease is indicated by the red triangle, which is 29 nucleotides upstream from the ATG start codon. (Bottom) confirmation of knockout by the WB analysis of U2OS WT (vector-transfected clone) or independent $P I N 1^{-/}$clones. (D) (Left) U2OS WT or PIN1 $1^{-/}$\#6 clones expressing Flag-FAAP20 were treated with $50 \mu \mathrm{g} /$ $\mathrm{mL}$ CHX for the indicated times, and degradation of Flag-FAAP20 was analyzed by WB. (Right) quantification of Flag-FAAP20 levels by ImageJ. A dotted line indicates the half-life of protein degradation. Data shown are the mean $\pm \mathrm{SD}$ from two independent experiments. ${ }^{*} P<0.001$ compared to WT, Student's t-test. (E) U2OS WT or PIN1 $1^{-/-}$cells transiently expressing Flag-FAAP20 were left untreated or treated with $10 \mu \mathrm{M}$ MG132 for 6 h, and 
cell lysates were analyzed by WB. (F, G) U2OS cells expressing Flag-FAAP20 WT or isomerization-defective mutants were treated with $50 \mu \mathrm{g} / \mathrm{mL}$ CHX for the indicated times and analyzed by WB. (H) U2OS cells stably expressing PIN1 WT or catalytically dead mutant (K63A/R68A/R69A) were transfected with Flag-FAAP20-encoding plasmid, and cell lysates were analyzed by WB. (I) 293T cells transfected with the indicated plasmids were treated with $20 \mu \mathrm{M}$ MG132 for $4 \mathrm{~h}$, lysed under denaturing conditions, and incubated with Ni-NTA agarose to capture polyubiquitinated Flag-FAAP20. Flag-FAAP20 in Figure 4 was transiently expressed by plasmid transfection.

https://doi.org/10.1371/journal.pgen.1007983.g004

(Fig 4C). Independent $\mathrm{KO}$ clones demonstrated that the half-life of FAAP20 degradation is dramatically shorter in the absence of PIN1 (Fig 4D and S4A Fig). Reduced levels of exogenous FAAP20 expression were rescued by proteasome inhibition, suggesting that PIN1 antagonizes FAAP20 degradation via the proteasome (Fig 4E and S4B Fig). Accordingly, mutations in the Ser48 or Pro49 residues of FAAP20 accelerated FAAP20 degradation, further supporting the idea that FAAP20 isomerization by PIN1 promotes FAAP20 stability (Fig 4F and 4G). The residues in the catalytic PPIase domain of PIN1, including Lys63, Arg68, and Arg69, form a positively charged phosphate-binding loop to coordinate the pSer/Thr of the substrate [35]. Importantly, reconstitution of the catalytically dead PIN1 mutant into $P I N 1^{-/}$cells failed to restore the FAAP20 levels reduced by PIN1 deletion, indicating that PIN1 activity is required for stabilizing cellular FAAP20 levels (Fig 4H and S4C Fig). Moreover, Ni-NTA pull-down of ubiquitinated proteins demonstrated that the polyubiquitination of FAAP20 increases in the FAAP20 P49A mutant and in the absence of PIN1, suggesting that FAAP20 is susceptible to degradation without PIN1-induced isomerization (Fig 4I and S4D Fig). Collectively, these data support the idea that PIN1 is required for maintaining FAAP20 stability.

\section{Protein phosphatase 2A dephosphorylates FAAP20 at the CPD degron motif}

Phosphorylation at the CPD of FAAP20 is prerequisite for FAAP20 degradation [17]. Thus, we next sought to determine the elements that control the FAAP20 degradation regulated by $\mathrm{CPD}$ phosphorylation and PIN1-induced isomerization. Protein phosphorylation is antagonized by phosphatases, and protein phosphatase 2A (PP2A) is a major proline-directed Ser/ $\mathrm{Thr}$ phosphatase known to regulate diverse cellular processes by counteracting kinase signaling [36]. PP2A has been shown to mediate dephosphorylation of several PIN1 substrates, preferentially recognizing a specific conformation [37]. Hence, we explored the possibility that PP2A is involved in regulating the FAAP20 phosphorylation at the CPD. We had previously generated a FAAP20 antibody that specifically recognizes the pS113 of the CPD, which was used to monitor the CPD phosphorylation status [17] (S5A Fig). Incubation of cells with the PP2A inhibitor okadaic acid (OA) [38] increased the pS113 of FAAP20 (Fig 5A). The PP2A holoenzyme exists as a heterotrimeric complex composed of a catalytic $\mathrm{C}$, a scaffolding $\mathrm{A}$, and a diverse group of regulatory $B$ subunits, which is further classified into four distinct families (B55, B56, $\mathrm{B}^{\prime \prime}$, and $\mathrm{B}^{\prime \prime \prime}$ ), each of which have several isoforms such as $\alpha, \beta, \gamma, \delta$, and $\varepsilon$ [36]. Specific knockdown of the PP2A catalytic subunit $\alpha$-isoform (PP2Ac; encoded by PPP2CA) with two independent siRNAs also elevated pS113 levels, indicating that the enzymatic activity of PP2A antagonizes FAAP20 phosphorylation at the CPD (Fig 5B and S5B Fig). Conversely, overexpression of PP2A was sufficient to decrease pS113 levels (Fig 5C).

To further explore the direct role of PP2A in FAAP20 dephosphorylation, we determined the interaction of FAAP20 with B56 $\alpha$, the $\alpha$-isoform of the largest B regulatory subunit family B56 ( $B^{\prime} /$ PR61), which interacts with a substrate and thus confers substrate specificity toward the PP2A holoenzyme [36]. Intriguingly, serial deletion mutagenesis of FAAP20 revealed that the N-terminal amino acid 1-30 residues are required for the interaction with B56 $\alpha$ (Fig 5D). As expected, the comparison between the FAAP20 $\Delta$ N30 and $\Delta$ N48 mutants showed that the 
A

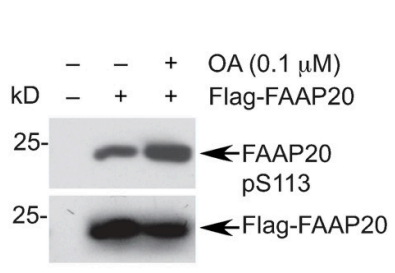

B

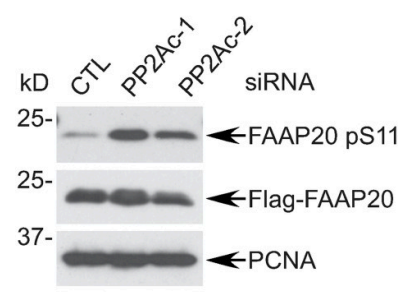

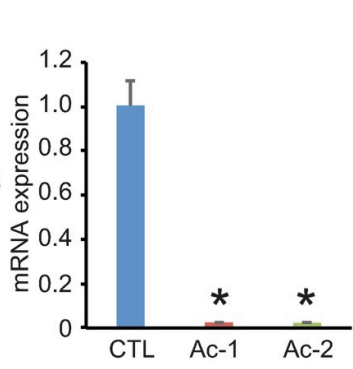

E
C

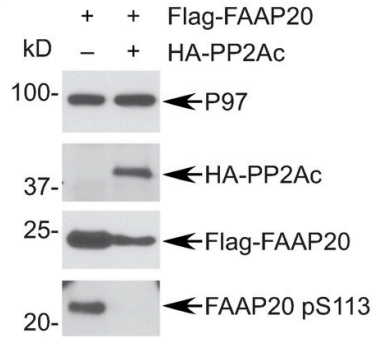

D
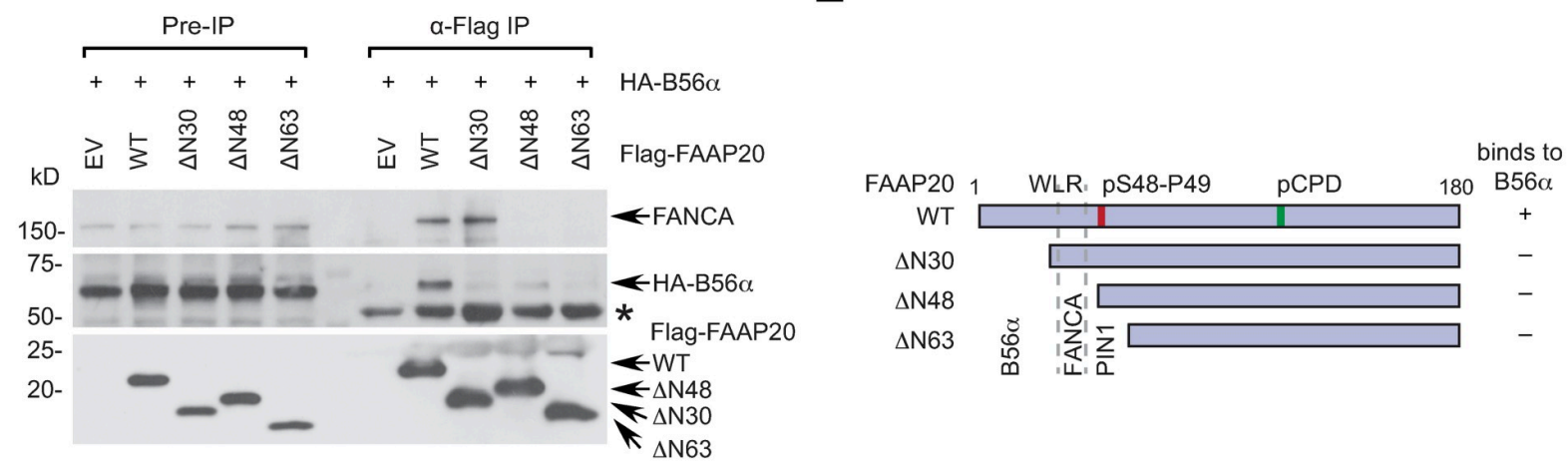

Fig 5. Protein phosphatase 2A dephosphorylates FAAP20 at the CPD motif. (A) U2OS cells expressing Flag-FAAP20 were left untreated or treated with $100 \mathrm{nM}$ okadaic acid (OA) for $2 \mathrm{~h}$, and FAAP20 pS113 levels were analyzed by WB using anti-pS113 antibody. (B) (Left) U2OS cells were serially transfected with siRNA control or PP2Ac and Flag-FAAP20-encoding plasmid, and FAAP20 pS113 levels were analyzed by WB. (Right) confirmation of PP2Ac knockdown by RT-qPCR. mRNA expression was normalized by GAPDH mRNA (mean \pm SD; $\mathrm{n}=3$ independent experiments). ${ }^{*} P<0.001$, Student's t-test. (C) U2OS cells were transfected with indicated plasmids, and FAAP20 pS113 levels were analyzed by WB. (D) $293 \mathrm{~T}$ cells transfected with HA-B56 $\alpha$ and Flag-FAAP20 WT or N-terminal deletion mutants ( $\Delta$ N30: a.a.1-30 deletion, $\Delta$ N48: a.a.1-48 deletion, and $\Delta$ N63: a.a.1-63 deletion) were subjected to anti-Flag IP and WB. * IgG heavy chain. (E) Summary of the binding regions for B56 $\alpha$, FANCA, and PIN1 revealed in this study.

https://doi.org/10.1371/journal.pgen.1007983.g005

amino acid 31-48 residues encompassing the WLR region were necessary for the interaction of FAAP20 with endogenous FANCA. Together, these data suggest that the N-terminal region of FAAP20 is a platform that serially mediates the interactions with multiple regulatory proteins involved in FAAP20 proteolysis, including B56 $\alpha$, FANCA, and PIN1 (Fig 5E). This also indicates that the affinity and activity of PP2A toward FAAP20 may be regulated by the FAAP20 isomerization, which occurs at the adjacent pSer-Pro motif by PIN1.

\section{PIN1-induced FAAP20 isomerization antagonizes the $\mathrm{SCF}^{\mathrm{FBW} 7}$-dependent ubiquitin signaling at the CPD motif}

Our results thus far raise the possibility that PIN1-mediated FAAP20 isomerization may increase the PP2A holoenzyme association with FAAP20, thereby promoting dephosphorylation of the CPD, which would prevent its interaction with FBW7 and subsequent degradation. Consistent with this idea, we observed that the FAAP20 WLR (i.e. isomerization-prone) mutant binds stronger to B56 $\alpha$ in comparison to WT (Fig 6A). On the other hand, the interaction of FAAP20 WT or mutant with GSK $\beta$ was largely unaffected, indicating that dephosphorylation may be a rate-limiting step for determining CPD phosphorylation status (S6A Fig). Regarding CPD phosphorylation, the FAAP20 WLR mutant exhibited lower pS113 levels compared to WT, and exogenous expression of PIN1 further decreased pS113 levels (Fig 6B).

Importantly, the ratio of pS113 signals in the upper and lower isoforms of FAAP20 was lower than that of the total FAAP20 immunoblot signal, and was further decreased following PIN1 
A

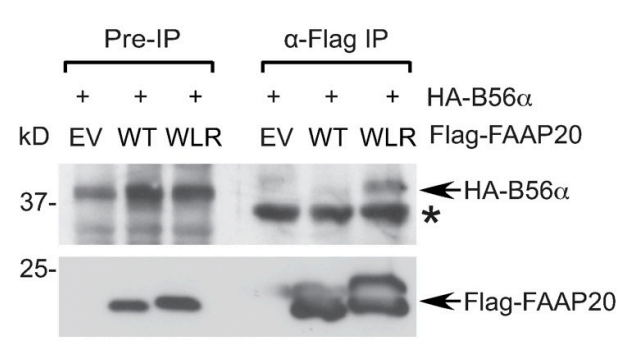

D

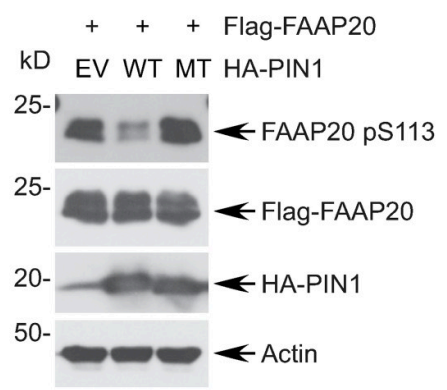

$\mathbf{F}$

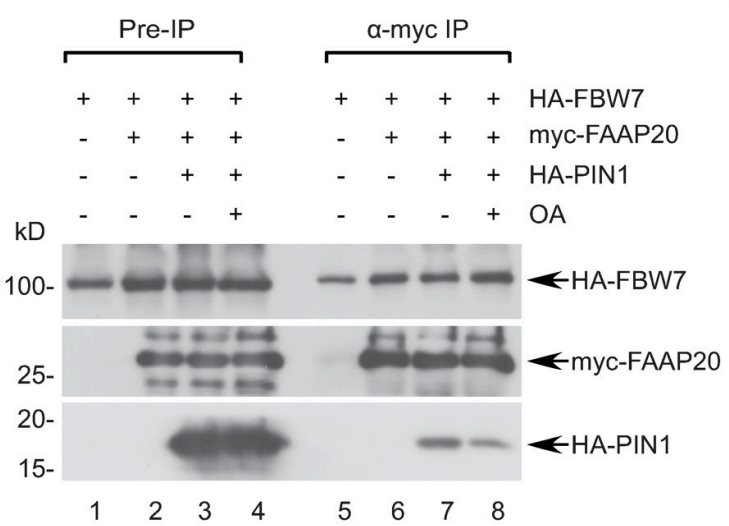

B

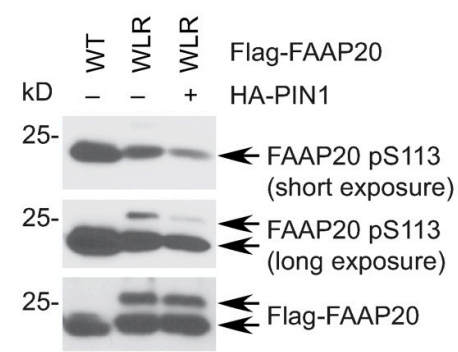

C

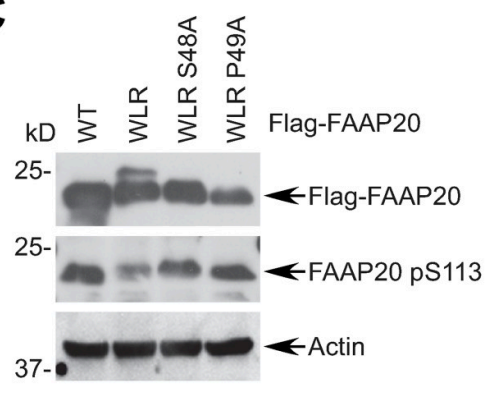

$\mathbf{E}$

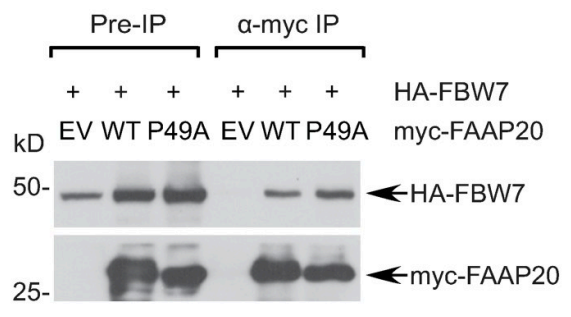

$\mathbf{G}$

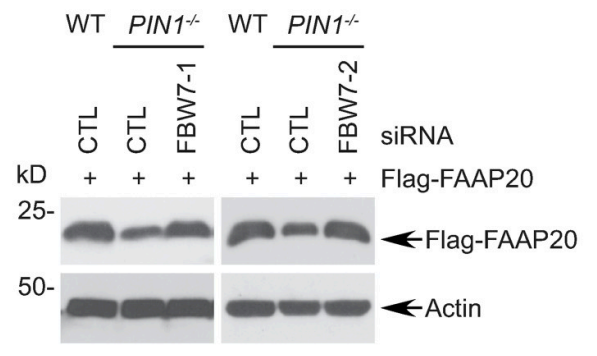

Fig 6. PIN1-induced FAAP20 isomerization promotes PP2A signaling and antagonizes FBW7-dependent FAAP20 degradation. (A) 293T cells were transfected with indicated plasmids, and the amount of HA-B56 $\alpha$ pulled-down by Flag-FAAP20 was analyzed by anti-Flag IP and WB. (B, C, D) U2OS cells were transfected with indicated plasmids, and pS113 levels of Flag-FAAP20 WT or various mutants were analyzed by WB. MT: K63A/ R68A/R69A mutant. (E) The amount of HA-FBW7 pulled-down by myc-FAAP20 WT or P49A was analyzed by anti-myc IP and WB of 293T cell lysates. (F) 293T cells were transfected with indicated plasmids, and the interaction between HA-FBW7 and myc-FAAP20 was analyzed by anti-myc coIP and WB. (-) denotes empty vector transfection. Where indicated, cells were treated with $50 \mathrm{nM}$ okadaic acid (OA) and $10 \mu \mathrm{M} \mathrm{MG132}$ for $6 \mathrm{~h}$ before harvest. (G) U2OS WT or PIN1 ${ }^{-/}$cells were serially transfected with two independent siRNA FBW7 oligos (vs. control) and Flag-FAAP20-encoding plasmid, and Flag-FAAP20 levels were analyzed by WB.

https://doi.org/10.1371/journal.pgen.1007983.g006

expression, indicating that the isomerized FAAP20 (i.e. upper isoform) is less prone to CPD phosphorylation, and because of its high affinity to PIN1, is more susceptible to dephosphorylation. This idea was further supported by the result showing that CPD phosphorylation was elevated when mutations were introduced to the pS48-p49 motif of the WLR mutant in comparison to the WLR mutation only (Fig 6C). Similarly, exogenous expression of PIN1 WT, but not the catalytically dead mutant, was sufficient to decrease the pS113 levels of WT FAAP20 (Fig 6D). Conversely, the isomerization-defective mutant, FAAP20 P49A, exhibited an increased interaction with FBW7, indicating that the increase in CPD phosphorylation, caused 
by reduction of PIN1 and PP2A activity, promotes $\mathrm{SCF}^{\mathrm{FBW} 7}$-dependent ubiquitin signaling (Fig 6E). To further support this idea, we examined the interplay among PIN1, PP2A, and FBW7 toward FAAP20 degradation. Co-immunoprecipitation demonstrated that exogenous expression of PIN1 decreased the interaction of FAAP20 with FBW7, which was restored by the inhibition of $\mathrm{PP} 2 \mathrm{~A}$ activity by $\mathrm{OA}$, indicating that $\mathrm{PIN} 1$ counteracts $\mathrm{SCF}^{\mathrm{FBW} 7}$ proteolytic signaling by promoting dephosphorylation of the CPD motif, mediated by PP2A (Fig 6F; compare FBW7 lanes $7 \& 8$ ). Accordingly, decreased FAAP20 levels in the absence of PIN1 were rescued by FBW7 depletion, suggesting that PIN1 restricts $\mathrm{SCF}^{\mathrm{FBW} 7}$ activity to promote FAAP20 stabilization (Fig 6G and S6B Fig). Collectively, these data support our model that PIN1-induced conformational change of FAAP20 promotes FAAP20 dephosphorylation at the $\mathrm{CPD}$ by $\mathrm{PP} 2 \mathrm{~A}$, thereby preventing $\mathrm{SCF}^{\mathrm{FBW}}$-dependent FAAP20 degradation.

\section{PIN1 promotes FANCD2 activation and DNA ICL repair}

Disruption of FAAP20 stability impairs the integrity of the FA complex, leading to a defect in FANCD2 activation required for the initiation of DNA ICL repair [13]. Thus, we hypothesized that PIN1 is an unidentified regulatory component of the FA core complex that controls FANCD2 monoubiquitination and examined the role of PIN1 in FA pathway signaling. PIN1 depletion using two independent siRNAs decreased the levels of FANCD2 monoubiquitination induced by a DNA cross-linking agent mitomycin C (MMC), which is visualized by the more slowly migrating, modified FANCD2 (FANCD2-Ub) in an immunoblot, indicating that PIN1 is required for promoting FANCD2 activation (Fig 7A). Under the prolonged treatment of cycloheximide, PIN1 knockdown resulted in accelerated degradation of FANCA, a direct interaction partner of FAAP20 in the FA core complex, which was antagonized by exogenous expression of the FAAP20 CPD mutant that is refractory to degradation by $\mathrm{SCF}^{\mathrm{FBW} 7}$ but proficient for FANCA interaction (S7A Fig). This suggests that defective FA pathway activation in PIN1-deficient cells results primarily from the compromised FA core complex caused by destabilization of FAAP20. Accordingly, cytometry-based quantification of $\gamma \mathrm{H} 2 \mathrm{AX}$, a marker for replication-associated DSBs, revealed that PIN1 knockdown significantly increases the cells with positive $\gamma \mathrm{H} 2 \mathrm{AX}$ signals upon MMC treatment when compared to control (Fig $7 \mathrm{~B}$ and 7C). Furthermore, a comet assay demonstrated that PIN1 depletion increases the levels of DNA breaks upon MMC treatment, together suggesting that defects in the FA pathway caused by PIN1 deficiency results in persistent DNA damage and impaired resolution of DNA lesions (Fig 7D). Accordingly, cells depleted of PIN1 were hypersensitive to MMC, indicating that PIN1 dictates the progression of DNA ICL repair and cellular survival (Fig 7E and S7B Fig). To further substantiate the specific role of FAAP20 isomerization in the FA pathway, we reconstituted siRNA-resistant FAAP20 WT or isomerization-defective mutants in FAA20-depleted cells and examined MMC sensitivity. While FAAP20 WT could complement MMC hypersensitivity of FAAP20-depleted cells, the FAAP20 S48A or P49A mutants failed to do so despite their comparable or higher expression than endogenous FAAP20, indicating that FAAP20 isomerization is required for the function of FAAP20 in the FA pathway (Fig 7F). On the other hand, the WLR mutant, which cannot interact with FANCA despite its increased stability, was not able to complement the FAAP20 deficiency (S7C Fig). The CPD mutant could not fully complement the FAAP20 deficiency either, since FANCA turnover dynamics during DNA ICL repair, which is regulated by FAAP20 phosphorylation and degradation, is also a determinant for DNA ICL repair outcome as previously described [17]. Together, the results of the WLR and CPD mutants further highlight the notion that the effect of PIN1-induced FAAP20 isomerization on DNA ICL repair is largely mediated through the FANCA interaction and the FA core complex. 
A

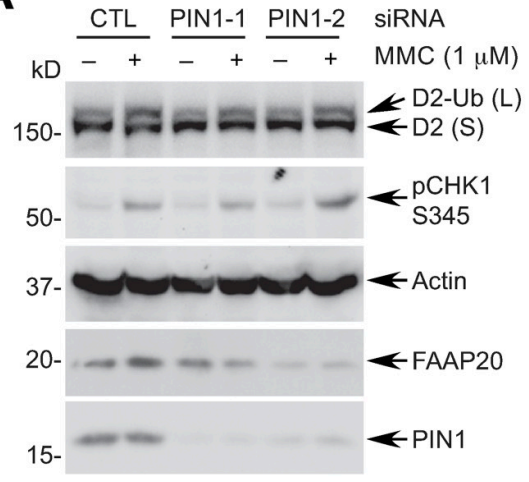

C

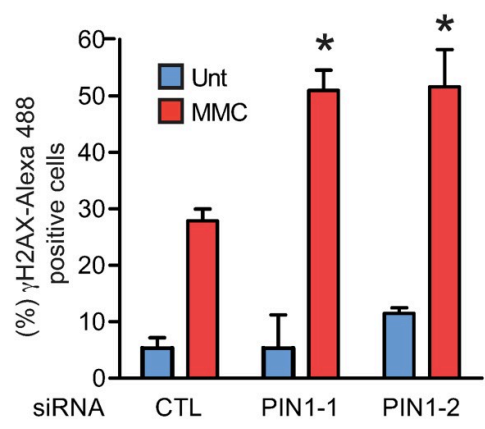

E

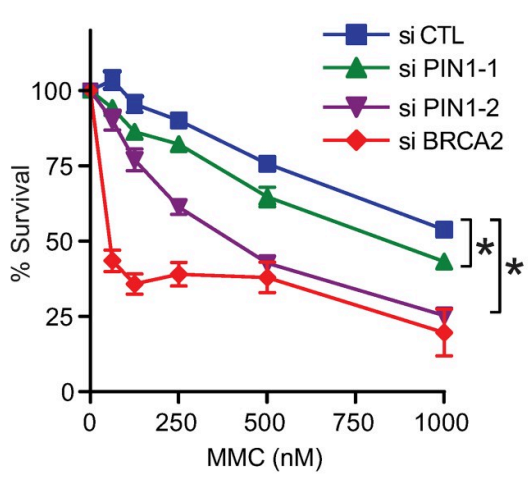

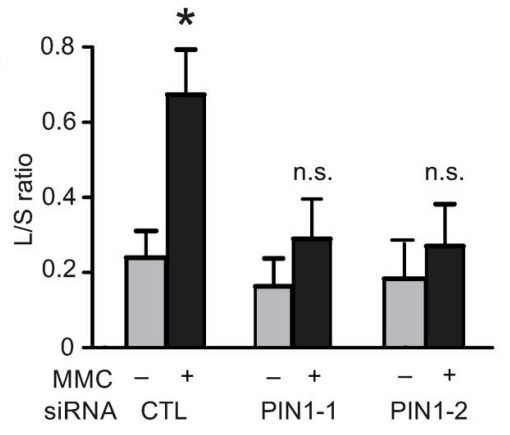

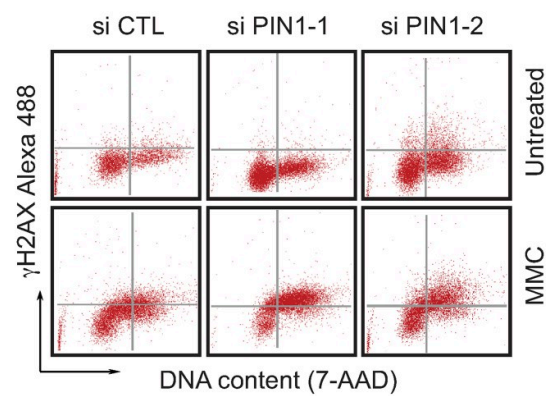

D
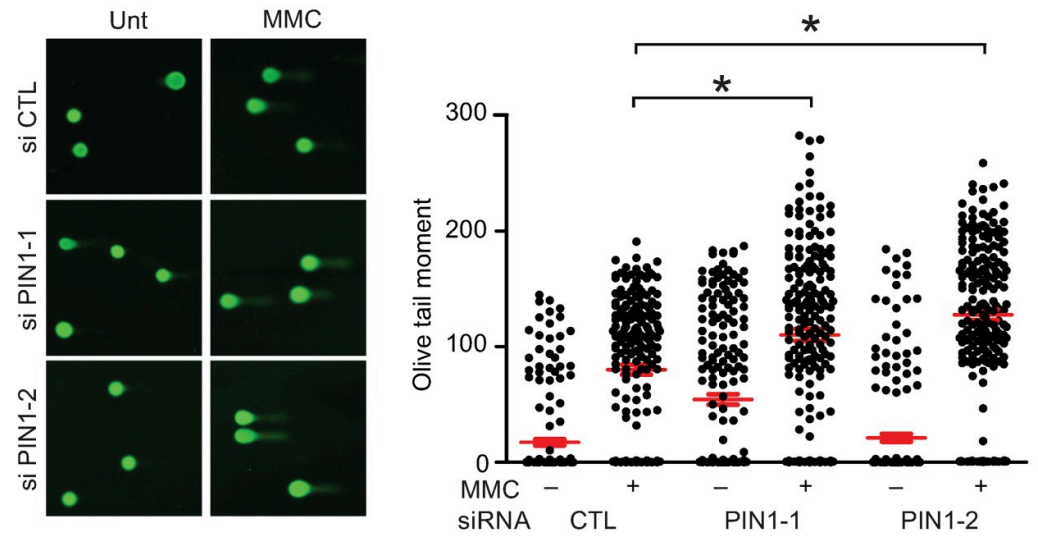

$\mathbf{F}$

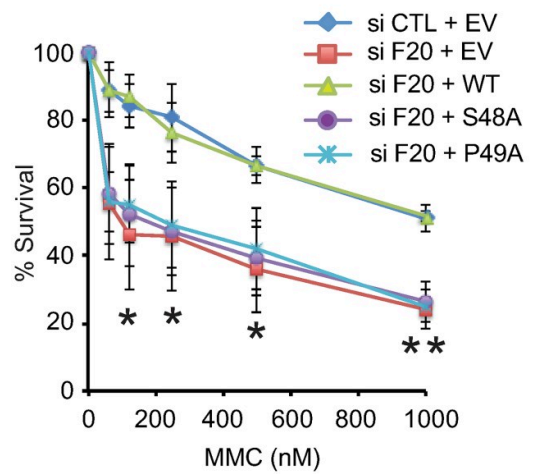

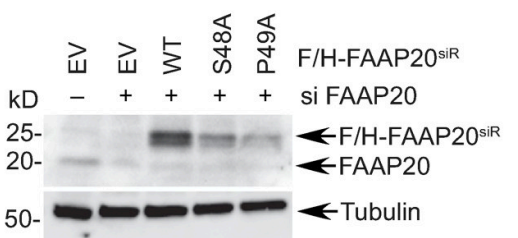

Fig 7. Knockdown of PIN1 impairs the FA pathway. (A) U2OS cells transfected with two independent PIN1 siRNAs (vs. control) were treated with $1 \mu \mathrm{M}$ mitomycin $\mathrm{C}(\mathrm{MMC})$ for $8 \mathrm{~h}$, and induction of FANCD2 monoubiquitination (FANCD2-Ub) was analyzed by WB. A representative image is shown, and FANCD2-Ub/FANCD2 (L/S) ratio was quantified by Image J from three independent experiments (right). Mean \pm SEM; ${ }^{*} P<0.05$ compared to untreated, two-tailed Student's t-test. n.s: not significant. (B) Representative flow cytometry of Alexa Fluor 488-conjugated $\gamma \mathrm{H} 2 \mathrm{AX}$ and 7-AAD staining from siRNA-transfected U2OS cells treated with $50 \mathrm{ng} / \mathrm{mL} \mathrm{MMC} \mathrm{for} 18 \mathrm{~h}$. Upper quadrants represent $\gamma \mathrm{H} 2 \mathrm{AX}$-positive cells. (C) Quantification of $\gamma \mathrm{H} 2 \mathrm{AX}$-positive cells. Error bar, mean $\pm \mathrm{SD} ; \mathrm{n}=3$ independent experiments, ${ }^{*} P<0.01$, Student's t-test. (D) (Left) a representative image of comet tails from siRNA-transfected U2OS cells treated with $0.3 \mu \mathrm{M} \mathrm{MMC} \mathrm{for} 18 \mathrm{~h}$. (Right) plotting of olive tail moment. Mean \pm SEM is shown in red. ${ }^{*} P<0.001$, two-tailed Student's t-test. Representative data from two independent experiments are shown. (E) Cell survival assay of U2OS cells transfected with indicated siRNA oligos. Data shown are mean \pm SEM from three independent experiments. ${ }^{*} P<0.05$, CTL vs. PIN1 knockdown, paired Student's t-test. (F) (Left) U2OS cells were sequentially transfected with siRNA FAAP20 and siRNA-resistant pMSCV-Flag-HA-FAAP20 WT or indicated mutants, and cellular viability was measured. Data shown are mean $\pm \mathrm{SD}$ from three independent experiments. ${ }^{*} P<0.05$, WT vs. S48A or P49A reconstitution, ${ }^{* *} P<0.01$, WT vs. S48A or P49A reconstitution, Student's t-test. (Right) WB analysis of reconstituted FAAP20 WT or mutants in FAAP20-depleted cells.

https://doi.org/10.1371/journal.pgen.1007983.g007 
A

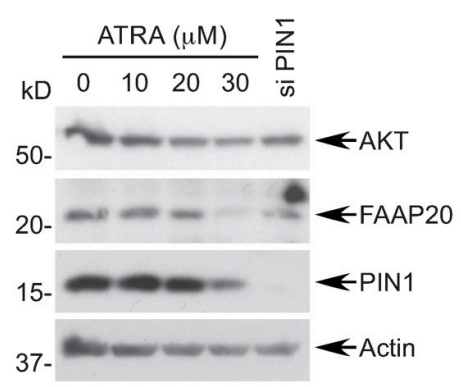

B

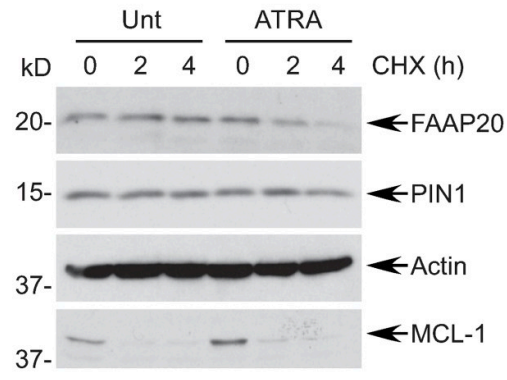

C

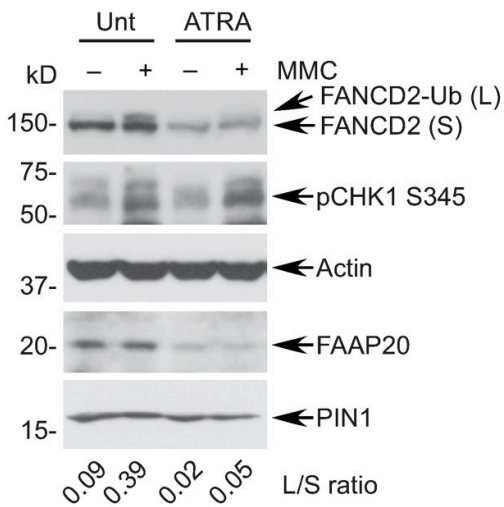

D

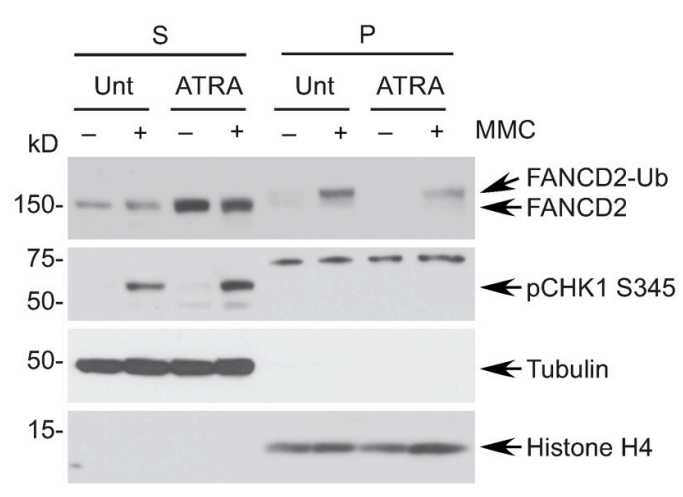

E

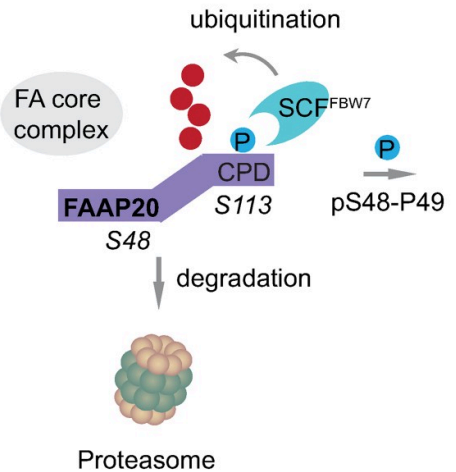

Prolyl cis-trans isomerization

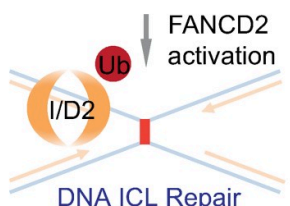

Fig 8. Pharmacological PIN1 inhibition disrupts FANCD2 activation. (A) MDA-MB-231 cells were treated with indicated doses of ATRA for $72 \mathrm{~h}$ and analyzed by WB with indicated antibodies. As a control, cells were transfected with siRNA PIN1 for $72 \mathrm{~h}$. (B) MDA-MB-231 cells treated with $30 \mu \mathrm{M}$ ATRA for $72 \mathrm{~h}$ were treated with $50 \mu \mathrm{g} / \mathrm{mL}$ CHX for the indicated times, and degradation of endogenous FAAP20 was analyzed by WB. (C) Cells treated with $30 \mu \mathrm{M}$ ATRA were challenged with $1 \mu \mathrm{M}$ MMC for $6 \mathrm{~h}$, and induction of FANCD2 monoubiquitination was analyzed by WB and quantitated by ImageJ. (D) ATRA and MMC-treated MDA-MB-231 cells were fractionated into cytosolic/nucleoplasmic (S) and chromatin-enriched (P) fractions, and chromatin association of FANCD2-Ub was analyzed by WB. Localization of tubulin and Histone H4 represents S and P fractions, respectively. (E) Model depicting the role of PIN1 in regulating the FA pathway. Phosphorylation of the CPD motif recruits $\mathrm{SCF}^{\mathrm{FBW} 7}$ and subjects FAAP20 for proteasomal degradation. PIN1-induced FAAP20 isomerization at the pS48-P49 motif enhances the interaction of FAAP20 with PP2A to dephosphorylate the $\mathrm{CPD}$ motif, thus antagonizing $\mathrm{SCF}^{\mathrm{FBW7}}$-mediated FAAP20 degradation. Stabilized FAAP20 in the FA core complex promotes FANCD2 activation and DNA ICL repair.

https://doi.org/10.1371/journal.pgen.1007983.g008

Modulation of FA pathway activity is closely associated with the chemotherapeutic efficacy of DNA cross-linking cytotoxic chemotherapy [39]. Thus, based on our findings, we determined whether pharmacological inhibition of PIN1 is sufficient to disrupt the FA pathway via FAAP20 destabilization in breast cancer, where high levels of PIN1 have been correlated with aggressiveness and chemoresistance [40]. Treatment of MDA-MB-231 triple negative breast cancer (TNBC) cell lines with the recently identified PIN1 inhibitor all-trans retinoic acid (ATRA), which binds to the PIN1 active site and degrades PIN1 [41], decreased the levels of FAAP20 in a dose-dependent manner, as well as the levels of AKT, a known target of PIN1, without significantly affecting cellular viability [42] (Fig 8A and S7D Fig). FAAP20 degradation was accelerated in the presence of ATRA, further confirming that PIN1 activity is required for FAAP20 stability (Fig 8B). Accordingly, MDA-MB-231 cells treated with ATRA exhibited less damage-induced FANCD2 monoubiquitination and reduced localization of monoubiquitinated FANCD2 to chromatin, indicating that signaling of FANCD2 activation by the FA core complex is disrupted (Fig $8 \mathrm{C}$ and $8 \mathrm{D}$ ). Collectively, these data allude to the potential of 
pharmacological PIN1 inhibition as a method for enhancing the chemotherapeutic response of cross-linking regimens by destabilizing FAAP20 and thus disrupting the FA pathway.

\section{Discussion}

\section{PIN1 as a new regulatory component of the FA pathway}

Despite the critical roles of PIN1 in regulating numerous cellular processes, its connection to DNA ICL repair and genome maintenance pathways has remained uncharacterized. Here, we have identified PIN1 as a new regulatory component of the FA core complex in the FA pathway and established the first direct link between PIN1-SCF ${ }^{\mathrm{FBW} 7}$-mediated proteolysis and DNA ICL repair. Our results propose a model wherein PIN1 maintains the integrity of the FA core complex via phosphorylation-dependent FAAP20 isomerization (Fig 8E). Dissociation of FAAP20 from FANCA in the FA core complex subjects FAAP20 to GSK3 $\beta$-dependent phosphorylation at the $\mathrm{CPD}$, leading to $\mathrm{SCF}^{\mathrm{FBW} 7}$-dependent polyubiquitination and proteasome delivery. PIN1 antagonizes this process by acting as a molecular switch to catalyze the isomerization of the phosphorylated S48-P49 motif of FAAP20 and induce its conformational change, which enhances its interaction with PP2A, subsequently decreasing CPD phosphorylation and $\mathrm{SCF}^{\mathrm{FBW}}$ interaction.

Importantly, our FAAP20 WLR mutant turned out to be a valuable separation-of-function mutant. It has lost its interaction with FANCA and is thus subject to degradation; however, it has also become more susceptible to PIN1-induced isomerization. This unique property allowed us to specifically address the PIN1-PP2A signaling that antagonizes $\mathrm{SCF}^{\mathrm{FBW}}$-dependent degradation of FAAP20 when dissociated from FANCA. Since the WLR-deletion mutant still strongly interacts with PIN1 and exhibits two isoforms, we do not believe that the WLR region directly mediates the PIN1 interaction (Fig 2E). Rather, the WLR region may be antagonistic for PIN1 access to the adjacent pS48-P49 motif, and disruption of this region may alter the local conformation of FAAP20, enhancing PIN1 targeting to FAAP20. We propose that the increased FAAP20 stability would allow FAAP20 to favorably associate with the FA core complex, thereby promoting the integrity of the FA core complex and FANCD2 activation upon damage. In other words, cellular levels of FAAP20 that are available to interact with FANCA, even the pool of FAAP20 that may transiently dissociate from FANCA, may be positively maintained by PIN1 in order to sustain FANCA and the FA core complex. PIN1-dependent FAAP20 isomerization may occur during translation before FAAP20 is incorporated into the FA core complex, and PIN1 counteracts the degradation process of FAAP20 to keep adequate levels of FAAP20 available for the interaction with FANCA. Alternatively, PIN1-induced isomerization also antagonizes degradation of FAAP20 while interacting with FANCA by preventing the access of $\mathrm{SCF}^{\mathrm{FBW}}$, thus further promoting the stability of the FA core complex. In this regard, PIN1 activity is critical for dictating the outcome of DNA ICL repair processes by modulating integrity of the FA core complex.

It is also tempting to speculate that PIN1-induced FAAP20 isomerization regulates the FAAP20-FANCA interaction dynamics during DNA ICL repair, and thus fine-tunes the activity of the FA core complex. We previously showed that FBW7 depletion alongside prolonged accumulation of FAAP20 impairs DNA ICL repair, indicating that spatiotemporal removal of FANCA-FAAP20, or the FA core complex as a whole, from DNA lesions is critical for the completion of DNA ICL repair [17]. Downregulation of PIN1 activity may trigger degradation of FAAP20, thus facilitating the clearance of the FA core complex to suppress FANCD2 monoubiquitination in the later stage of repair. Indeed, various post-translational modifications of PIN1, including phosphorylation and SUMOylation, affect PIN1 activity, and PIN1 is known to be phosphorylated in a DNA damage-dependent manner [35,43-46]. Thus, elucidating how 
modification of PIN1 in response to the DNA damage checkpoint regulates PIN1 activity will be an important future direction to more clearly understand how PIN1 contributes to genomic integrity. Interestingly, we observed that the P49A mutation of FAAP20 does not disrupt its interaction with FANCA, indicating that conformational change of FAAP20 by isomerization per se does not directly regulate the FANCA interaction (S7E Fig). In contrast, we consistently notice that the S48A mutation more or less impairs FANCA interaction, indicating that phosphorylation of FAAP20 may regulate its association with the FA core complex independently of isomerization, although its exact role remains to be determined.

\section{Mechanisms of PIN1 regulation on phosphorylation-dependent ubiquitin signaling}

Our study provides important mechanistic insights into how phosphorylation-dependent ubiquitin-proteasome signaling is regulated by PIN1-catalyzed isomerization. Here, we show that PIN1 accelerates conformational changes of phosphorylated FAAP20, which affects its interaction with regulatory proteins, including PP2A and FBW7, thus changing the fate of the protein. This result highlights a complex interplay among prolyl isomerization, phosphorylation, and ubiquitin signaling, which is in agreement with previous studies of known PIN1 substrates. Proline-directed phosphatase PP2A is conformation-specific, and PIN1-induced prolyl isomerization is known to allow PP2A to interact with and dephosphorylate the pSer/Thr-Pro motif of Cdc25C [37]. PIN1 also interacts with the pThr231-Pro motif of tau, which facilitates PP2A-dependent dephosphorylation of hyperphosphorylated tau, restoring its function in microtubule assembly [37,47]. Moreover, PIN1-induced isomerization at the pThr58-Pro motif of c-Myc has been shown to enhance PP2A function to dephosphorylate Ser62, which promotes c-Myc degradation [25]. These studies support the notion that PIN1-induced isomerization is an important regulatory mechanism for controlling PP2A-mediated protein dephosphorylation, which determines the kinetics of substrate degradation and modulates its function. Interestingly, a recent study reported that conformational changes of the epigenetic modulator BRD4 by PIN1 not only prevent its degradation, but also increase its interaction with the downstream transcriptional regulator CDK9 and thus BRD4's transcriptional activity [48]. By directly visualizing the CPD phosphorylation status that dictates FAAP20 degradation, we were able to provide a mechanism in which a conformational change of FAAP20 by PIN1 modulates the dynamic interaction between PP2A and FBW7 with FAAP20. Currently, it is not clear how a specific conformation of FAAP20 favors the interaction with PP2A or whether PIN1-induced isomerization at the pS48-P49 motif also influences the conformational change at the CPD. Interestingly, we showed that a defined N-terminal region of FAAP20 preceding the pS48-p49 motif is responsible for interacting with the substrate-binding subunit of PP2A and FANCA. This suggests that a local conformational change at the pS48-P49 motif by PIN1 can readily influence the association of FAAP20 with PP2A, although we do not exclude the possibility of FANCA that binds FAAP20 through the WLR region directly affecting the interaction of FAAP20 to PP2A. A detailed structural analysis of this region would be required to reveal how PIN1-induced isomerization affects the dynamic interactions of FAAP20 with its regulatory proteins.

\section{Exploiting the FA pathway via PIN1 inhibition for cancer therapy}

Besides to the pathogenesis of FA, the mechanistic principle developed in our studies has important clinical implications to cancer, since exploiting deregulation of PIN1 activity in the FA pathway could alter the response of cancer cells to cytotoxic chemotherapy or poly ADPribose polymerase (PARP) inhibitors [49]. Numerous efforts have been made to develop small 
molecule inhibitors to modulate reversible monoubiquitination of FANCD2 and inhibit the FA pathway, thereby augmenting the sensitivity of cancer cells to cytotoxic chemotherapy regimens, including platinum $[12,50,51]$. Intriguingly, PIN1 is widely overexpressed in many human cancers and is associated with poor clinical outcomes [52,53]. In particular, increased activity is often observed in the majority of human breast cancers, and PIN1 is considered to be an essential factor for breast tumorigenesis, as well as cancer stem cells [24,40,54,55]. Accordingly, PIN1 inhibition has been considered as an attractive strategy for cancer therapy [56]. Our data indicate that PIN1 inhibition and subsequent disruption of the FA pathway can potentially function as a chemosensitizer for DNA cross-linking cytotoxic chemotherapy. This may be particularly relevant to the TNBC subtype of breast cancer, which shares similar molecular features to the tumors arising from BRCA1/FANCS and BRCA2/FANCD1-associated DNA repair deregulation [57]. A recent study also proposed a role of PIN1 in suppressing CtIP, and thus homologous recombination (HR), which may increase error-prone repair and promote tumorigenesis, indicating that PIN1 inhibition could be a general strategy to supplement chemosensitization or exploit the synthetic lethality of PARP inhibition in PIN1-upregulated tumors [28]. Future studies to characterize a comprehensive regulatory network that governs PIN1-PP2A-SCF ${ }^{\mathrm{FBW} 7}$ signaling will provide important mechanistic insights into the proteolytic control of the FA pathway in preserving genomic integrity and allow for the development of therapeutic strategies to exploit aberrant DNA repair in cancer cells caused by deregulated phosphorylation-dependent ubiquitin signaling.

\section{Materials and methods}

\section{Cell culture and plasmid construction}

U2OS and 293T cell lines were acquired from the American Tissue Culture Collection (ATCC). MDA-MB-231 was a kind gift from Jun Chung (Stony Brook Medicine). Cells were cultured in Dulbecco's Modified Eagle's Medium supplemented with 10\% fetal bovine serum and $1 \%$ penicillin/streptomycin, following standard culture conditions and procedures. FAAP20, FBW7, and GSK3 $\beta$ constructs were previously described [17]. Plasmids encoding GST-PIN1 was a gift from Michael Yaffe (Addgene plasmid \#19027), His-PIN1 from Dustin Maly (Addgene plasmid \#40773), V245 pCEP-4HA-B56 $\alpha$ from David Virshup (Addgene plasmid \#14532), and pBABE-zeo PPP2CA from William Hahn (Addgene plasmid \#10689). PIN1 cDNA was subcloned into modified pcDNA3-HA or pMSCV-Flag-HA vectors (Invitrogen). Point or deletion mutations were introduced using the QuikChange II XL Site-Directed Mutagenesis (SDM) kit (Agilent Technologies) and confirmed by DNA sequencing (SBU DNA sequencing facility). Stable cell lines were generated by retroviral transduction of pMSCVFlag-HA-PIN1 constructs using $8 \mu \mathrm{g} / \mathrm{mL}$ polybrene (Sigma-Aldrich), followed by selection with $2 \mu \mathrm{g} / \mathrm{mL}$ puromycin. Viruses were generated from 293T cells that were co-transfected with pMSCV-Flag-HA-PIN1, pCMV-Gag/Pol and pCMV-VSV-G.

\section{Plasmid and siRNA transfection}

Transient plasmid transfection was performed using GeneJuice (Millipore) according to the manufacturer's protocols. siRNA duplexes were transfected at $25 \mathrm{nM}$ using Lipofectamine RNAiMAX (Invitrogen). The following DNA sequences were targeted by siRNA: Control: $5^{\prime}$ CAGGGTATCGACGATTACAAA-3'; FAAP20: $5^{\prime}$-CACGGTGAGCCCGGAGCTGAT-3'; PIN1-1: 5'-CGGCTACATCCAGAAGATCAA-3'; PIN1-2: 5'-CAGGCCGAGTGTACTACT TCA-3'; FBW7-1: 5'-GTGGAATGCAGAGACTGGAGA-3'; FBW7-2: 5'-CGGGTGAATTTA TTCGAAATT-3'; BRCA2: 5' -TTGAAGAATGCAGGTTTAATA-3' (Qiagen). siRNA sequences for hPP2Ac are $5^{\prime}$-GAACTTGACGATACTCTAAtt-3' (\#1; s10959) and 5'-CCAAA 
CUAUUGUUAUCGUUtt-3' (\#2; s10957) and synthesized from Ambion, Thermo Fisher. Generation of siRNA-resistant FAAP20 was previously described [58].

\section{Antibodies and chemicals}

Antibodies used in this study included: FAAP20 (HPA038829, Sigma-Aldrich), Flag (F1804, Sigma-Aldrich), c-Myc (9E10, Sigma-Aldrich), $\alpha$-Tubulin (sc-8035, Sigma-Aldrich), FANCD2 (FI-17, Santa Cruz), PCNA (PC-10, Santa Cruz), PIN1 (A302-316A, Bethyl), FANCA (A301980A, Bethyl), MCL-1 (A302-715A, Bethyl), $\gamma$-Tubulin (A302-631A, Bethyl), HA (6E2, Cell Signaling), ubiquitin (P4D1, Cell Signaling), $\beta$-Actin (4967, Cell Signaling), p97 (2648, Cell Signaling), pCHK1 S345 (2341, Cell Signaling), $\gamma \mathrm{H} 2$ AX (2577, Cell Signaling), AKT (9272, Cell Signaling), Histone H4 (07-108, Millipore), BRCA2 (OP95, Millipore), and pS113 FAAP20 (in house; Genscript). Mitomycin C (M5030), Z-Leu-Leu-Leu-al (MG132; C2211), and cycloheximide (C4859), were purchased from Sigma-Aldrich. Okadaic acid (459620) was from EMD Millipore and all-trans retinoic acid (ATRA)/Tretinoin (S1653) was from Selleckchem. Drugs were used at the concentrations indicated in the figure legends.

\section{Western blotting, immunoprecipitation, and subcellular fractionation}

Cells were lysed in NETN300 buffer (1\% NP40, $300 \mathrm{mM} \mathrm{NaCl}, 0.1 \mathrm{mM}$ EDTA, and $50 \mathrm{mM}$ Tris [pH 7.5]) supplemented with protease inhibitor cocktail (Roche) and halt phosphatase inhibitor cocktail (Thermo Fisher), resolved by SDS-PAGE, transferred onto PVDF membranes (Millipore), and antibodies were detected using an enhanced chemiluminescence method. Some of immunoblot images were acquired by iBright CL1000 imaging system (Thermo Fisher). For co-immunoprecipitation, 293T cells were lysed in NETN150 buffer (1\% NP40, $150 \mathrm{mM} \mathrm{NaCl}, 0.1 \mathrm{mM}$ EDTA, and $50 \mathrm{mM}$ Tris [pH 7.5]) in the presence of protease and phosphatase inhibitor cocktails and were centrifuged at 15,000 rpm for $10 \mathrm{~min}$ at $4{ }^{\circ} \mathrm{C}$. Cell lysates were incubated with anti-Flag M2 affinity gel (A2220, Sigma-Aldrich) or anti-c-Myc agarose affinity gel (A7470, Sigma-Aldrich) for $4 \mathrm{~h}$ followed by five washes with NETN150 buffer. Resins were boiled in 2X Laemmli sample buffer and subjected to SDS-PAGE. Subcellular fractionation was performed as previously described [59]. Briefly, cells were lysed using cytoskeleton (CSK) buffer (10 mM Tris [pH 6.8], $100 \mathrm{mM} \mathrm{NaCl}, 300 \mathrm{mM}$ sucrose, $3 \mathrm{mM} \mathrm{MgCl}_{2}, 1 \mathrm{mM}$ EGTA, $1 \mathrm{mM} \mathrm{EDTA}$, and $0.1 \%$ Triton X-100) for $5 \mathrm{~min}$ on ice. After centrifugation at $1,500 \mathrm{~g}$ for $5 \mathrm{~min}$, the supernatant (S) was separated from the pellet (P), and pellets were sequentially lysed in PBS and $2 \mathrm{X}$ boiling lysis buffer (50 $\mathrm{mM}$ Tris [pH 6.8], $2 \%$ SDS, and $850 \mathrm{mM} \beta$-mercaptoethanol).

\section{Mass spectrometry}

After separation via SDS-PAGE and coomassie blue staining, excised gel pieces were destained, reduced, alkylated, and digested with trypsin gold (Promega, V5280), essentially as previously described with minor modifications [60]. The resulting peptide extract was dried and dissolved in a solution of $2 \%$ acetonitrile (ACN), $0.1 \%$ formic Acid (FA) (buffer A) for analysis by automated microcapillary liquid chromatography-tandem mass spectrometry. Fused-silica capillaries $\left(100 \mu \mathrm{m}\right.$ inner diameter-i.d.) were pulled using a P-2000 $\mathrm{CO}_{2}$ laser puller (Sutter Instruments, Novato, CA) and packed with $10 \mathrm{~cm}$ of $5 \mu \mathrm{m}$ ProntoSil 120-5-C18H (Bischoff Chromatography, Leonberg, Germany) using a pressure bomb. The samples were loaded via an Eksigent NanoLC Autosampler. The column was installed in-line with an Eksigent Nano2D High Performance Liquid Chromatography (HPLC) pump running at $300 \mathrm{~nL} \mathrm{~min}{ }^{-1}$. The peptides were eluted from the column by applying a 115 min gradient from $2 \%$ buffer $B$ ( $98 \% \mathrm{ACN}, 0.1 \% \mathrm{FA}$ ) to $40 \%$ buffer B. The gradient was switched from $40 \%$ to $80 \%$ buffer B 
over $3 \mathrm{~min}$ and held constant for $3 \mathrm{~min}$. Finally, the gradient was changed from $80 \%$ buffer B to $2 \%$ buffer B over $0.1 \mathrm{~min}$, and then held constant at $2 \%$ buffer B for 29 more minutes. The application of a $2.2 \mathrm{kV}$ distal voltage electrosprayed the eluting peptides directly into an LTQ Orbitrap XL ion trap mass spectrometer (Thermo Fisher) equipped with a nano-liquid chromatography electrospray ionization source. Full mass spectra (MS) were recorded on the peptides over a 400 to $2000 \mathrm{~m} / z$ range at 60,000 resolution, followed by top-five MS/MS scans in the ion-trap. Charge state dependent screening was turned on, and peptides with a charge state of +2 or higher were analyzed. Mass spectrometer scan functions and HPLC solvent gradients were controlled by the Xcalibur data system (Thermo Fisher). MS/MS spectra were extracted from the RAW file with ReAdW.exe (http://sourceforge.net/projects/sashimi). The resulting mzXML data files were searched with The GPM X!Tandem and MaXQuant Andromeda search engines against a custom database composed of the Uniprot human proteome with added sequences for common contaminants.

\section{NMR spectroscopy}

The non-phosphorylated and phosphorylated FAAP20 peptides were synthesized from Genscript. All NMR experiments were performed on a Bruker $850 \mathrm{MHZ}$ Avance III spectrometer at $25^{\circ} \mathrm{C}$ equipped with a cryoprobe. NMR samples contained $2 \mathrm{mM}$ peptide in $10 \mathrm{mM}$ sodium phosphate $\mathrm{pH} 6.5$, and $10 \% \mathrm{D}_{2} \mathrm{O}$, in the absence or presence of $0.03 \mathrm{mM}$ PIN1. Total Correlation Spectroscopy (TOCSY) data for the peptide in the absence of PIN1 was collected with 4096 (TDF2) and 256 data points (TDF1), a spectral width of $10 \mathrm{ppm}(8503 \mathrm{~Hz}) \times 10 \mathrm{ppm}$ $(8503 \mathrm{~Hz}), 80 \mathrm{~ms}$ mixing time, and $10417 \mathrm{~Hz}$ spinlock frequency. ROESY data for the peptide in the presence of PIN1 were acquired with similar data points and spectral width as described for the TOCSY experiment but with different mixing times of 30, 50, 70, 90, $110 \mathrm{~ms}$, and 4310 $\mathrm{Hz}$ spinlock frequency. ROESY data for the substrate peptide in the presence and absence of PIN1 were also acquired with $300 \mathrm{~ms}$ mixing time. All NMR spectra were processed with Topspin and analyzed with CcpNmr Analysis. The $\mathrm{I}_{\mathrm{tc}} / \mathrm{I}_{\mathrm{tt}}$ ratios (peak intensity ratio of the conformational exchange trans-to-cis cross-peak to the trans conformation diagonal-peak) for pSer7 and Glu9, which precedes and follows Pro8 respectively, depend on the forward $\left(\mathrm{K}_{\mathrm{ct}}{ }^{\mathrm{cat}}\right)$ and reverse $\left(\mathrm{K}_{\mathrm{tc}}{ }^{\mathrm{cat}}\right)$ rate constants for the two-state cis-to-trans conformational exchange process. In order to determine $\mathrm{K}_{\mathrm{ct}}{ }^{\mathrm{cat}}$ and $\mathrm{K}_{\mathrm{tc}}{ }^{\mathrm{c}}$, $\mathrm{tc} / \mathrm{tt}$ ratios were fitted to the equation below using KaleidaGraph (Synergy software). $\mathrm{I}_{\mathrm{tc}} / \mathrm{I}_{\mathrm{tt}}=\mathrm{K}_{\mathrm{tc}}{ }^{\mathrm{cat}}\left[\exp \left(\mathrm{K}_{\mathrm{ex}} \mathrm{t}_{\mathrm{m}}\right)-1\right] /\left[\mathrm{K}_{\mathrm{ct}}{ }^{\mathrm{cat}} \exp \left(\mathrm{K}_{\mathrm{ex}} \mathrm{t}_{\mathrm{m}}\right)+\mathrm{K}_{\mathrm{tc}}{ }^{\mathrm{cat}}\right]$, where $t_{m}$ is the mixing time, while $K_{e x}$ is the sum of $K_{c t}{ }^{c a t}$ and $K_{t c}{ }^{c a t}$

\section{Protein purification and GST pull-down assay}

GST pull-down was performed as previously described [59]. Briefly, for the interaction between GST-PIN1 and Flag-FAAP20 in vitro, GST or GST-PIN1 was expressed using E. coli BL21 (DE3) expression strain induced with $0.5 \mathrm{mM}$ isopropyl $\beta$-D-1-thiogalactopyranoside (IPTG, Sigma-Aldrich) at $30^{\circ} \mathrm{C}$. Cells were lysed in PBS with lysozyme, sonicated, and further incubated with $1 \%$ Triton X-100. Cell lysates were recovered by centrifugation at 15,000 rpm at $4{ }^{\circ} \mathrm{C}$ for $15 \mathrm{~min}$ and incubated with glutathione-sepharose beads (GE Healthcare). After washing, the beads were incubated with in vitro transcribed and translated (IVTT) proteins in NETN150 buffer for $3 \mathrm{~h}$ at $4{ }^{\circ} \mathrm{C}$ followed by three washes. For IVTT, a total of $250 \mathrm{ng}$ of pcDNA3 plasmids were incubated with $10 \mu \mathrm{L}$ of TnT T7 Quick Coupled Transcription/Translation Master Mix (Promega) at $30{ }^{\circ} \mathrm{C}$ for 70 min to produce proteins. For the purification of recombinant PIN1 for NMR analysis, His-PIN1 was expressed using BL21 (DE3) cells in 2xYT medium with $0.5 \mathrm{mM}$ IPTG at $18{ }^{\circ} \mathrm{C}$ overnight. Cells were resuspended and crushed in NiA buffer (20 mM Tris [pH 7.5], $500 \mathrm{mM} \mathrm{NaCl}, 5 \%$ glycerol, and $5 \mathrm{mM}$ imidazole) using the 
EmulsiFlex-C3 homogenizer (Avestin). The soluble protein was loaded onto Ni-NTA resin, washed, and eluted with $\mathrm{NiB}$ buffer (20 mM Tris [pH 7.5], $500 \mathrm{mM} \mathrm{NaCl}, 5 \%$ glycerol, and 1 $\mathrm{M}$ imidazole). The eluate was dialyzed against $20 \mathrm{mM}$ Tris [pH 7.5], $500 \mathrm{mM} \mathrm{NaCl}, 5 \%$ glycerol, and stored at $-80{ }^{\circ} \mathrm{C}$.

\section{In vivo ubiquitin assay}

In vivo ubiquitin assays were performed under denaturing conditions. MG132-treated cells were resuspended with PBS/1\% SDS, snap-frozen in liquid nitrogen, and boiled for $15 \mathrm{~min}$. Cell lysates were diluted 10-fold with PBS and centrifuged at $15,000 \mathrm{rpm}$ for $15 \mathrm{~min}$ at $4{ }^{\circ} \mathrm{C}$. Lysate aliquots (4\%) were saved for input, and lysates were incubated with HisPur Ni-NTA Resin (Thermo Fisher) in the presence of $10 \mathrm{mM}$ imidazole (Sigma-Aldrich) at $4{ }^{\circ} \mathrm{C}$ for $3 \mathrm{~h}$, followed by five washes with PBS/0.1\% SDS, $10 \mathrm{mM}$ imidazole. Resins were boiled in 2X Laemmli sample buffer and subjected to SDS-PAGE and Western blotting.

\section{RT-qPCR}

RNA was isolated using TRIzol (Invitrogen). cDNA synthesis was performed using a highcapacity cDNA reverse transcription kit (Applied Biosystems) according to the manufacturer's protocols. Real-time quantitative PCR was performed using Fast SYBR Green Master Mix (Applied Biosystems) and a StepOnePlus Real-Time PCR system (Applied Biosystems). GAPDH mRNA levels were used as a control for normalization. The following primers were used for cDNA amplification: PP2Ac forward 5'-CAGCTAGTGATGGAGGGATA-3'; PP2Ac reverse 5'-TGGGTCAAACTGCAAGAAA-3'; FBW7 forward 5'-CACTCAAAGT GTG GAATGCAGAGAC-3'; FBW7 reverse 5'-GCATCTCGAGAACCGCTAACAA-3'; GAPDH forward 5'-CAACTACATGGTTTACATGTTC-3'; GAPDH reverse 5'- GCCAGTGGACTCC ACGAC- $3^{\prime}$.

\section{CRISPR/Cas9 gene editing}

A pair of oligos containing the human PIN1 sgRNA targeting sequence was designed using crispr.mit.edu. The forward oligo sequence is $5^{\prime}$-CACCGATGCGCTTCTCCCAGCCGGG-3' and the reverse oligo sequence is $5^{\prime}$-AAACCCCGGCTGGGAGAAGCGCATC- ${ }^{\prime}$. Annealed oligos were cloned into pSpCas9(BB)-2A-Puro (pX459; a gift from Feng Zhang, Addgene plasmid \#48139) and transfected into U2OS cells using GeneJuice (Millipore). Control cells were transfected with a pX459 empty vector. Twenty-four hours after transfection, cells were selected with $2 \mu \mathrm{g} / \mathrm{mL}$ puromycin. After recovery from selection, cells were seeded onto 96-well plates, in medium without puromycin, for clonal selection. Selected clones were subsequently analyzed by Western blotting, using anti-PIN1 antibody, to confirm successful knockout.

\section{Cell survival assay}

Cells in 6-well plates were transfected with siRNA oligos and seeded on 96-well plates the next day. Cells were treated with increasing doses of MMC in duplicates at $48 \mathrm{~h}$ after transfection, and cell viability was determined using the CellTiter-Glo luminescent cell viability assay (Promega) 4-5 days after continuous drug treatment. Luminescence was measured using a GloMax Navigator microplate luminometer (Promega). Mean values were analyzed for statistical significance using paired Student's t-test. 


\begin{abstract}
Alkaline comet assay
Single-cell gel electrophoresis for the detection of MMC-induced DNA breaks was performed using the CometAssay kit (4250-050-K, Trevigen) according to the manufacturer's protocol. Twenty-five $\mu \mathrm{L}$ of a cell suspension at $2 \times 10^{5}$ cells per $\mathrm{mL}$ were combined with $225 \mu \mathrm{L}$ of low-melting agarose (1:10 ratio, vol/vol), and $50 \mu \mathrm{L}$ were spread on Comet slides (Trevigen). After solidification, the slides were immersed in cold lysing solution at $4{ }^{\circ} \mathrm{C}$ for $45 \mathrm{~min}$ and placed in freshly prepared alkaline unwinding solution $(200 \mathrm{mM} \mathrm{NaOH}, 1 \mathrm{mM}$ EDTA) for $20 \mathrm{~min}$ at RT. Electrophoresis of unwound DNA was performed at $21 \mathrm{~V}$ for 30 $\mathrm{min}$. The slides were washed with $\mathrm{dH} 2 \mathrm{O}$ for $5 \mathrm{~min}$, dehydrated with $70 \%$ ethanol for $5 \mathrm{~min}$, dried, and stained with SYBR Gold (Thermo Fisher). Comet tails were examined using a Nikon Eclipse E600 fluorescence microscope and analyzed by OpenComet [61]. Per group, up to 300 individual nuclei were evaluated. The olive tail moment was calculated as a measure of DNA damage and presented as the product of the DNA \% (tail intensity) and the distance between the intensity-weighted centroids of a head and a tail (DNA migration). Difference between mean values was tested for statistical significance using two-tailed unpaired Student's t-test.
\end{abstract}

\title{
Flow cytometry
}

For quantification of $\gamma \mathrm{H} 2 \mathrm{AX}$-positive cells, cell pellets were pre-extracted with $\mathrm{PBS} / 0.5 \%$ Triton-X for $5 \mathrm{~min}$, fixed with $4 \%$ paraformaldehyde for $15 \mathrm{~min}$, and incubated with $\gamma \mathrm{H} 2 \mathrm{AX}$ Alexa Fluor 488 (1:100; CR55T33, Thermo Fisher) in Foxp3/transcription factor staining buffer (Thermo Fisher) for $1 \mathrm{~h}$. Cells were washed once and suspended in $500 \mu \mathrm{L}$ 7-AAD viability staining solution (Thermo Fisher) supplemented with $200 \mu \mathrm{g} / \mathrm{mL}$ PureLink RNase A, stained for $30 \mathrm{~min}$ at $37^{\circ} \mathrm{C}$, and analyzed by Attune NxT acoustic focusing cytomether and Attune NxT software v2.7 (Thermo Fisher).

\section{Statistical analysis}

Student's t-test was used to assess the statistical significance of our results, using Prism (GraphPad).

\section{Supporting information}

S1 Fig. Characterization of the FAAP20 WLR by mass spectrometry. (A) Silver staining and Western blotting (WB) of Flag-FAAP20 WT and WLR mutant purified from 293T cells by anti-Flag immunoprecipitation (IP) and elution with Flag peptide. The Flag-FAAP20 WLR mutant exhibits an additional slower migrating isoform (U) alongside the isoform observed in the WT (L). (B) Mass spectrometry (MS) analysis of the purified Flag-FAAP20 WLR isoforms. Both upper and lower bands from Flag-FAAP20 WLR were excised and analyzed by MS. The pSer48 residue shaded in blue is specifically present in the upper isoform of Flag-FAAP20 WLR. (C) Annotated MS/MS spectrum for 39-69 peptide of the FAAP20 WLR mutant phosphorylated at Ser48. The sequence of the peptide is displayed horizontally and in the righthand vertical panel. The phosphorylated serine is indicated by the lower case ' $\mathrm{s}$ ' in red at residue 48 . The $y$ and $b$ ions annotated in the figure are indicated by the encircled ion number in the right-hand vertical sequence. Evidence that phosphorylation occurs at Ser48 is supported by the strong $\mathrm{y}_{22}{ }^{2+}$ spectral peak $\left({ }^{*}\right)$ and the identified y ion series surrounding this ion. (D) (Top) $293 \mathrm{~T}$ cells transiently transfected with indicated plasmids were analyzed by WB. Immunoblots were quantitated by ImageJ, and the U/L ratio was derived from the average of 
two independent experiments. (Bottom) U2OS cells were serially transfected with PIN1 siRNA (vs. control) and Flag-FAAP20 WLR, and lysates were analyzed by WB.

(TIF)

S2 Fig. Interaction between PIN1 and FAAP20. (A) Lysates from 293T cells were incubated with glutathione beads bound with GST or GST-PIN1 and the levels of precipitated endogenous FAAP20 was analyzed by WB. (B) In vitro transcribed and translated (IVTT) FAAP20 WT, WLR point or deletion mutants were immunoprecipitated by anti-Flag agarose and analyzed by WB.

(TIF)

S3 Fig. Analysis of the pFAAP20 peptide isomerization rate catalyzed by PIN1. (A) Shown are the ratios of cross-peak and diagonal-peak intensities $\left(\mathrm{I}_{\mathrm{tc}} / \mathrm{I}_{\mathrm{tt}}\right)$ for the trans-to-cis conformational change of pSer7 and Glu9 over increasing mixing time as well as its isomerization rate $\left(\mathrm{K}_{\mathrm{tc}}{ }^{\mathrm{cat}}\right)$. For the determination of $\mathrm{K}_{\mathrm{ct}}{ }^{\mathrm{cat}}$ and $\mathrm{K}_{\mathrm{tc}}{ }^{\mathrm{cat}}$, $\mathrm{tc} / \mathrm{tt}$ ratios were fitted to the equation given in the Materials and Methods. (B) Mean values of the cis-to-trans isomerization rate $\left(\mathrm{K}_{\mathrm{tc}}{ }^{\mathrm{cat}}\right.$ and $\mathrm{K}_{\mathrm{ct}}{ }^{\mathrm{cat}}$ ) of pSer7 and Glu9 are indicated. The cis-to-trans conformational exchange rate is enhanced 8.72-fold $\left(\mathrm{K}_{\mathrm{ct}}{ }^{\mathrm{cat}} / \mathrm{K}_{\mathrm{tc}}{ }^{\mathrm{cat}}=8.72\right)$.

(TIF)

S4 Fig. PIN1 knockout promotes FAAP20 degradation. (A) U2OS WT or PIN1 $1^{-/-} \# 1$ clones expressing Flag-FAAP20 were treated with $50 \mu \mathrm{g} / \mathrm{mL}$ CHX for the indicated times and degradation of Flag-FAAP20 was analyzed by WB. (B) Quantification of Flag-FAAP20 levels of Fig $4 \mathrm{E} \mathrm{PIN1} 1^{-/-}$\#6 from two independent experiments. ${ }^{*} p<0.01$, unpaired two-tailed t-test. (C) Quantification of Flag-FAAP20 levels of Fig $4 \mathrm{H}$ from two independent experiments. ${ }^{*} p<0.05$, unpaired two-tailed t-test. (D) U2OS WT or PIN1 ${ }^{-1-}$ \#6 clones cells transfected with the indicated plasmids were treated with $10 \mu \mathrm{M}$ MG132 for $6 \mathrm{~h}$, lysed under denaturing conditions, and incubated with Ni-NTA agarose to capture polyubiquitinated Flag-FAAP20.

(TIF)

S5 Fig. Confirmation of antibody and siRNA. (A) 293T cells expressing Flag-FAAP20 wildtype, S113A/S117A, or S48A mutant were treated with $10 \mu \mathrm{M}$ MG132 for $4 \mathrm{~h}$ and pS113 levels were analyzed by WB. (B) U2OS cells serially transfected with siRNA PP2Ac-1 and -2 (vs. control) and HA-PP2Ac-encoding plasmid were analyzed by anti-HA WB to confirm the specific targeting of siRNA PP2Ac to PP2Ac cDNA.

(TIF)

S6 Fig. The FAAP20-GSKß interaction and confirmation of knockdown. (A) 293T cells were transfected with indicated plasmids, and the amount of HA-GSK $\beta$ pulled-down by FlagFAAP20 was analyzed by anti-Flag IP and WB. (B) Confirmation of FBW7 knockdown by RT-qPCR. mRNA expression was normalized by GAPDH mRNA (mean $\pm \mathrm{SD} ; \mathrm{n}=2$ independent experiments of duplicated samples), ${ }^{*} P<0.001$, Student's t-test.

(TIF)

S7 Fig. Characterization of the PIN1-depleted cells. (A) U2OS cells serially transfected with siRNA PIN1 (vs. control) and Flag-FAAP20 CPD (S113A \& S117A) (vs. EV) were treated with $100 \mu \mathrm{g} / \mathrm{mL}$ CHX for the indicated times, and cell lysates were analyzed by WB. A short-lived protein MCL-1 serves as a control for CHX treatment. Endogenous FANCA levels were quantified using ImageJ from two independent experiments. (B) U2OS cells transfected with indicated siRNA oligos were analyzed by WB. (C) (Left) WB analysis of U2OS cells depleted of FAAP20 and reconstituted with siRNA-resistant pMSCV-Flag-HA (F/H)-tagged FAAP20 WT, $\triangle$ WLR (a.a.40-45 deletion), or CPD (S113A \& S117A). (Right) cellular viability of U2OS 
cells reconstituted as above. Data shown are mean \pm SEM from three independent experiments. ${ }^{*} P<0.05$, WT vs. WLR reconstitution, paired two-tailed Student's t-test. (D) The viability of MDA-MB-231 cells treated with indicated concentration of ATRA for $72 \mathrm{~h}$ was determined by luminescence-based quantification of cellular ATP levels. Mean \pm SD; $n=3$ independent experiments, n.s. not significant, Student's t-test. (E) 293T cells transiently transfected with indicated Flag-FAAP20 plasmids were subjected to Flag IP, and co-immunoprecipitated endogenous FANCA was analyzed by WB.

(TIF)

\section{Acknowledgments}

We would like to thank Alexandra Weinheimer for editorial assistance and Alan D. D'Andrea (Dana-Farber Cancer Institute) for critical reading of the manuscript. We thank Dr. Jun Chung (Stony Brook Medicine) for providing cell lines and Dr. Kun Ping Lu (Harvard Medical School) for reagents.

\section{Author Contributions}

Conceptualization: Jingming Wang, Hyungjin Kim.

Data curation: Jingming Wang, Bryan Chan, Michael Tong, Ukhyun Jo, Dwight Martin, Markus Seeliger, John Haley, Hyungjin Kim.

Formal analysis: Jingming Wang, Bryan Chan, Michael Tong, Ukhyun Jo, Dwight Martin, Markus Seeliger, John Haley, Hyungjin Kim.

Funding acquisition: Markus Seeliger, Hyungjin Kim.

Investigation: Jingming Wang, Bryan Chan, Michael Tong, YiTing Paung, Ukhyun Jo, Dwight Martin, Markus Seeliger, John Haley, Hyungjin Kim.

Methodology: Jingming Wang, Michael Tong, Ukhyun Jo, Dwight Martin, Hyungjin Kim.

Supervision: Hyungjin Kim.

Validation: Hyungjin Kim.

Writing - original draft: Michael Tong, Dwight Martin, Hyungjin Kim.

Writing - review \& editing: Jingming Wang, Michael Tong, Hyungjin Kim.

\section{References}

1. Ceccaldi R, Sarangi P, D'Andrea AD (2016) The Fanconi anaemia pathway: new players and new functions. Nat Rev Mol Cell Biol 17: 337-349. https://doi.org/10.1038/nrm.2016.48 PMID: 27145721

2. Deans AJ, West SC (2011) DNA interstrand crosslink repair and cancer. Nat Rev Cancer 11: 467-480. https://doi.org/10.1038/nrc3088 PMID: 21701511

3. D'Andrea AD (2010) Susceptibility pathways in Fanconi's anemia and breast cancer. N Engl J Med 362: 1909-1919. https://doi.org/10.1056/NEJMra0809889 PMID: 20484397

4. Garaycoechea JI, Patel KJ (2014) Why does the bone marrow fail in Fanconi anemia? Blood 123: 2634. https://doi.org/10.1182/blood-2013-09-427740 PMID: 24200684

5. Kim H, D'Andrea AD (2012) Regulation of DNA cross-link repair by the Fanconi anemia/BRCA pathway. Genes Dev 26: 1393-1408. https://doi.org/10.1101/gad.195248.112 PMID: 22751496

6. Kottemann MC, Smogorzewska A (2013) Fanconi anaemia and the repair of Watson and Crick DNA crosslinks. Nature 493: 356-363. https://doi.org/10.1038/nature11863 PMID: 23325218

7. Lachaud C, Moreno A, Marchesi F, Toth R, Blow JJ, et al. (2016) Ubiquitinated Fancd2 recruits Fan1 to stalled replication forks to prevent genome instability. Science 351: 846-849. https://doi.org/10.1126/ science.aad5634 PMID: 26797144 
8. Schlacher $\mathrm{K}, \mathrm{Wu} \mathrm{H}$, Jasin M (2012) A distinct replication fork protection pathway connects Fanconi anemia tumor suppressors to RAD51-BRCA1/2. Cancer Cell 22: 106-116. https://doi.org/10.1016/j.ccr. 2012.05.015 PMID: 22789542

9. Rajendra E, Oestergaard VH, Langevin F, Wang M, Dornan GL, et al. (2014) The genetic and biochemical basis of FANCD2 monoubiquitination. Mol Cell 54: 858-869. https://doi.org/10.1016/j.molcel.2014. 05.001 PMID: 24905007

10. van Twest S, Murphy VJ, Hodson C, Tan W, Swuec P, et al. (2017) Mechanism of Ubiquitination and Deubiquitination in the Fanconi Anemia Pathway. Mol Cell 65: 247-259. https://doi.org/10.1016/j. molcel.2016.11.005 PMID: 27986371

11. Huang Y, Leung JW, Lowery M, Matsushita N, Wang Y, et al. (2014) Modularized functions of the Fanconi anemia core complex. Cell Rep 7: 1849-1857. https://doi.org/10.1016/j.celrep.2014.04.029 PMID: 24910428

12. Jo U, Kim H (2015) Exploiting the Fanconi Anemia Pathway for Targeted Anti-Cancer Therapy. Mol Cells 38: 669-676. https://doi.org/10.14348/molcells.2015.0175 PMID: 26194820

13. Kim H, Yang K, Dejsuphong D, D'Andrea AD (2012) Regulation of Rev1 by the Fanconi anemia core complex. Nat Struct Mol Biol 19: 164-170. https://doi.org/10.1038/nsmb.2222 PMID: 22266823

14. Leung JW, Wang Y, Fong KW, Huen MS, Li L, et al. (2012) Fanconi anemia (FA) binding protein FAAP20 stabilizes FA complementation group A (FANCA) and participates in interstrand cross-link repair. Proc Natl Acad Sci U S A 109: 4491-4496. https://doi.org/10.1073/pnas.1118720109 PMID: 22396592

15. Ali AM, Pradhan A, Singh TR, Du C, Li J, et al. (2012) FAAP20: a novel ubiquitin-binding FA nuclear core-complex protein required for functional integrity of the FA-BRCA DNA repair pathway. Blood 119: 3285-3294. https://doi.org/10.1182/blood-2011-10-385963 PMID: 22343915

16. Xie J, Kim H, Moreau LA, Puhalla S, Garber J, et al. (2015) RNF4-mediated polyubiquitination regulates the Fanconi anemia/BRCA pathway. J Clin Invest 125: 1523-1532. https://doi.org/10.1172/JCI79325 PMID: 25751062

17. Wang J, Jo U, Joo SY, Kim H (2016) FBW7 regulates DNA interstrand cross-link repair by modulating FAAP20 degradation. Oncotarget https://doi.org/10.18632/oncotarget.9595 PMID: 27232758

18. Yaffe MB, Schutkowski M, Shen M, Zhou XZ, Stukenberg PT, et al. (1997) Sequence-specific and phosphorylation-dependent proline isomerization: a potential mitotic regulatory mechanism. Science 278: 1957-1960. PMID: 9395400

19. Liou YC, Zhou XZ, Lu KP (2011) Prolyl isomerase Pin1 as a molecular switch to determine the fate of phosphoproteins. Trends Biochem Sci 36: 501-514. https://doi.org/10.1016/j.tibs.2011.07.001 PMID: 21852138

20. Ding Q, Huo L, Yang JY, Xia W, Wei Y, et al. (2008) Down-regulation of myeloid cell leukemia-1 through inhibiting Erk/Pin 1 pathway by sorafenib facilitates chemosensitization in breast cancer. Cancer Res 68: 6109-6117. https://doi.org/10.1158/0008-5472.CAN-08-0579 PMID: 18676833

21. Pulikkan JA, Dengler V, Peer Zada AA, Kawasaki A, Geletu M, et al. (2010) Elevated PIN1 expression by C/EBPalpha-p30 blocks C/EBPalpha-induced granulocytic differentiation through c-Jun in AML. Leukemia 24: 914-923. https://doi.org/10.1038/leu.2010.37 PMID: 20376080

22. van Drogen F, Sangfelt O, Malyukova A, Matskova L, Yeh E, et al. (2006) Ubiquitylation of cyclin E requires the sequential function of SCF complexes containing distinct hCdc4 isoforms. Mol Cell 23: 3748. https://doi.org/10.1016/j.molcel.2006.05.020 PMID: 16818231

23. Welcker M, Orian A, Jin J, Grim JE, Harper JW, et al. (2004) The Fbw7 tumor suppressor regulates glycogen synthase kinase 3 phosphorylation-dependent c-Myc protein degradation. Proc Natl Acad Sci U S A 101: 9085-9090. https://doi.org/10.1073/pnas.0402770101 PMID: 15150404

24. Wulf GM, Ryo A, Wulf GG, Lee SW, Niu T, et al. (2001) Pin1 is overexpressed in breast cancer and cooperates with Ras signaling in increasing the transcriptional activity of c-Jun towards cyclin D1. EMBO J 20: 3459-3472. https://doi.org/10.1093/emboj/20.13.3459 PMID: 11432833

25. Yeh E, Cunningham M, Arnold H, Chasse D, Monteith T, et al. (2004) A signalling pathway controlling cMyc degradation that impacts oncogenic transformation of human cells. Nat Cell Biol 6: 308-318. https://doi.org/10.1038/ncb1110 PMID: 15048125

26. Zacchi P, Gostissa M, Uchida T, Salvagno C, Avolio F, et al. (2002) The prolyl isomerase Pin1 reveals a mechanism to control p53 functions after genotoxic insults. Nature 419: 853-857. https://doi.org/10 1038/nature01120 PMID: 12397362

27. Zheng H, You H, Zhou XZ, Murray SA, Uchida T, et al. (2002) The prolyl isomerase Pin1 is a regulator of p53 in genotoxic response. Nature 419: 849-853. https://doi.org/10.1038/nature01116 PMID: 12397361 
28. Steger M, Murina O, Huhn D, Ferretti LP, Walser R, et al. (2013) Prolyl isomerase PIN1 regulates DNA double-strand break repair by counteracting DNA end resection. Mol Cell 50: 333-343. https://doi.org/ 10.1016/j.molcel.2013.03.023 PMID: 23623683

29. Hilton BA, Li Z, Musich PR, Wang H, Cartwright BM, et al. (2015) ATR Plays a Direct Antiapoptotic Role at Mitochondria, which Is Regulated by Prolyl Isomerase Pin1. Mol Cell 60: 35-46. https://doi.org/10. 1016/j.molcel.2015.08.008 PMID: 26387736

30. Min SH, Lau AW, Lee TH, Inuzuka H, Wei S, et al. (2012) Negative regulation of the stability and tumor suppressor function of Fbw7 by the Pin1 prolyl isomerase. Mol Cell 46: 771-783. https://doi.org/10. 1016/j.molcel.2012.04.012 PMID: 22608923

31. Bax A, Davis DG (1985) Practical aspects of two-dimensional transverse NOE spectroscopy. Journal of Magnetic Resonance (1969) 63: 207-213.

32. Pastorino L, Sun A, Lu PJ, Zhou XZ, Balastik M, et al. (2006) The prolyl isomerase Pin1 regulates amyloid precursor protein processing and amyloid-beta production. Nature 440: 528-534. https://doi.org/ 10.1038/nature04543 PMID: 16554819

33. Wang Y, Liu C, Yang D, Yu H, Liou YC (2010) Pin1At encoding a peptidyl-prolyl cis/trans isomerase regulates flowering time in Arabidopsis. Mol Cell 37: 112-122. https://doi.org/10.1016/j.molcel.2009.12. 020 PMID: 20129060

34. Zhou W, Yang Q, Low CB, Karthik BC, Wang Y, et al. (2009) Pin1 catalyzes conformational changes of Thr-187 in p27Kip1 and mediates its stability through a polyubiquitination process. J Biol Chem 284 : 23980-23988. https://doi.org/10.1074/jbc.M109.022814 PMID: 19584057

35. Lee TH, Chen $\mathrm{CH}$, Suizu F, Huang $\mathrm{P}$, Schiene-Fischer $\mathrm{C}$, et al. (2011) Death-associated protein kinase 1 phosphorylates Pin1 and inhibits its prolyl isomerase activity and cellular function. Mol Cell 42: 147159. https://doi.org/10.1016/j.molcel.2011.03.005 PMID: 21497122

36. Seshacharyulu P, Pandey P, Datta K, Batra SK (2013) Phosphatase: PP2A structural importance, regulation and its aberrant expression in cancer. Cancer Lett 335: 9-18. https://doi.org/10.1016/j.canlet. 2013.02.036 PMID: 23454242

37. Zhou XZ, Kops O, Werner A, Lu PJ, Shen M, et al. (2000) Pin1-dependent prolyl isomerization regulates dephosphorylation of Cdc25C and tau proteins. Mol Cell 6: 873-883. PMID: 11090625

38. Cohen P, Klumpp S, Schelling DL (1989) An improved procedure for identifying and quantitating protein phosphatases in mammalian tissues. FEBS Lett 250: 596-600. PMID: 2546812

39. Clauson C, Scharer OD, Niedernhofer L (2013) Advances in understanding the complex mechanisms of DNA interstrand cross-link repair. Cold Spring Harb Perspect Biol 5: a012732. https://doi.org/10. 1101/cshperspect.a012732 PMID: 24086043

40. Wulf G, Ryo A, Liou YC, Lu KP (2003) The prolyl isomerase Pin1 in breast development and cancer. Breast Cancer Res 5: 76-82. PMID: 12631385

41. Wei S, Kozono S, Kats L, Nechama M, Li W, et al. (2015) Active Pin1 is a key target of all-trans retinoic acid in acute promyelocytic leukemia and breast cancer. Nat Med 21: 457-466. https://doi.org/10. 1038/nm.3839 PMID: 25849135

42. Liao Y, Wei Y, Zhou X, Yang JY, Dai C, et al. (2009) Peptidyl-prolyl cis/trans isomerase Pin1 is critical for the regulation of PKB/Akt stability and activation phosphorylation. Oncogene 28: 2436-2445. https://doi.org/10.1038/onc.2009.98 PMID: 19448664

43. Chen $\mathrm{CH}$, Chang CC, Lee TH, Luo M, Huang P, et al. (2013) SENP1 deSUMOylates and regulates Pin1 protein activity and cellular function. Cancer Res 73: 3951-3962. https://doi.org/10.1158/00085472.CAN-12-4360 PMID: 23633483

44. Lee YC, Que J, Chen YC, Lin JT, Liou YC, et al. (2013) Pin1 acts as a negative regulator of the G2/M transition by interacting with the Aurora-A-Bora complex. J Cell Sci 126: 4862-4872. https://doi.org/10. 1242/jcs.121368 PMID: 23970419

45. Lu PJ, Zhou XZ, Liou YC, Noel JP, Lu KP (2002) Critical role of WW domain phosphorylation in regulating phosphoserine binding activity and Pin1 function. J Biol Chem 277: 2381-2384. https://doi.org/10. 1074/jbc.C100228200 PMID: 11723108

46. Matsuoka S, Ballif BA, Smogorzewska A, McDonald ER 3rd, Hurov KE, et al. (2007) ATM and ATR substrate analysis reveals extensive protein networks responsive to DNA damage. Science 316: 11601166. https://doi.org/10.1126/science.1140321 PMID: 17525332

47. Lu P-J, Wulf G, Zhou XZ, Davies P, Lu KP (1999) The prolyl isomerase Pin1 restores the function of Alzheimer-associated phosphorylated tau protein. Nature 399: 784. https://doi.org/10.1038/21650 PMID: 10391244

48. Hu X, Dong SH, Chen J, Zhou XZ, Chen R, et al. (2017) Prolyl isomerase PIN1 regulates the stability, transcriptional activity and oncogenic potential of BRD4. Oncogene 36: 5177-5188. https://doi.org/10. 1038/onc.2017.137 PMID: 28481868 
49. Lord CJ, Ashworth A (2017) PARP inhibitors: Synthetic lethality in the clinic. Science 355: 1152-1158. https://doi.org/10.1126/science.aam7344 PMID: 28302823

50. Jacquemont $C$, Taniguchi $T$ (2007) Proteasome function is required for DNA damage response and fanconi anemia pathway activation. Cancer Res 67: 7395-7405. https://doi.org/10.1158/0008-5472.CAN07-1015 PMID: 17671210

51. Liang Q, Dexheimer TS, Zhang P, Rosenthal AS, Villamil MA, et al. (2014) A selective USP1-UAF1 inhibitor links deubiquitination to DNA damage responses. Nat Chem Biol 10: 298-304. https://doi.org/ 10.1038/nchembio.1455 PMID: 24531842

52. Bao L, Kimzey A, Sauter G, Sowadski JM, Lu KP, et al. (2004) Prevalent overexpression of prolyl isomerase Pin1 in human cancers. Am J Pathol 164: 1727-1737. https://doi.org/10.1016/S0002-9440(10) 63731-5 PMID: 15111319

53. Lu Z, Hunter T (2014) Prolyl isomerase Pin1 in cancer. Cell Res 24: 1033-1049. https://doi.org/10. 1038/cr.2014.109 PMID: 25124924

54. Girardini JE, Napoli M, Piazza S, Rustighi A, Marotta C, et al. (2011) A Pin1/mutant p53 axis promotes aggressiveness in breast cancer. Cancer Cell 20: 79-91. https://doi.org/10.1016/j.ccr.2011.06.004 PMID: 21741598

55. Rustighi A, Zannini A, Tiberi L, Sommaggio R, Piazza S, et al. (2014) Prolyl-isomerase Pin1 controls normal and cancer stem cells of the breast. EMBO Molecular Medicine 6: 99-119. https://doi.org/10. 1002/emmm.201302909 PMID: 24357640

56. Zhou XZ, Lu KP (2016) The isomerase PIN1 controls numerous cancer-driving pathways and is a unique drug target. Nat Rev Cancer.

57. Oonk AM, van Rijn C, Smits MM, Mulder L, Laddach N, et al. (2012) Clinical correlates of 'BRCAness' in triple-negative breast cancer of patients receiving adjuvant chemotherapy. Ann Oncol 23: 2301-2305. https://doi.org/10.1093/annonc/mdr621 PMID: 22357256

58. Wojtaszek JL, Wang S, Kim H, Wu Q, D’Andrea AD, et al. (2014) Ubiquitin recognition by FAAP20 expands the complex interface beyond the canonical UBZ domain. Nucleic Acids Res 42: 1399714005. https://doi.org/10.1093/nar/gku1153 PMID: 25414354

59. Jo U, Cai W, Wang J, Kwon Y, D'Andrea AD, et al. (2016) PCNA-Dependent Cleavage and Degradation of SDE2 Regulates Response to Replication Stress. PLoS Genet 12: e1006465. https://doi.org/10. 1371/journal.pgen.1006465 PMID: 27906959

60. Shevchenko A, Wilm M, Vorm O, Mann M (1996) Mass spectrometric sequencing of proteins silverstained polyacrylamide gels. Anal Chem 68: 850-858. PMID: 8779443

61. Gyori BM, Venkatachalam G, Thiagarajan PS, Hsu D, Clement M-V (2014) OpenComet: An automated tool for comet assay image analysis. Redox Biology 2: 457-465. https://doi.org/10.1016/j.redox.2013. 12.020 PMID: 24624335 